\title{
Search for Signatures of Extra Dimensions in the Diphoton Mass Spectrum at the Large Hadron Collider
}

\author{
S. Chatrchyan et al.* \\ (CMS Collaboration) \\ (Received 4 December 2011; published 12 March 2012)
}

\begin{abstract}
A search for signatures of extra spatial dimensions in the diphoton invariant-mass spectrum has been performed with the CMS detector at the LHC. No excess of events above the standard model expectation is observed using a data sample collected in proton-proton collisions at $\sqrt{s}=7 \mathrm{TeV}$ corresponding to an integrated luminosity of $2.2 \mathrm{fb}^{-1}$. In the context of the large-extra-dimensions model, lower limits are set on the effective Planck scale in the range of $2.3-3.8 \mathrm{TeV}$ at the $95 \%$ confidence level. These limits are the most restrictive bounds on virtual-graviton exchange to date. The most restrictive lower limits to date are also set on the mass of the first graviton excitation in the Randall-Sundrum model in the range of 0.86-1.84 TeV, for values of the associated coupling parameter between 0.01 and 0.10 .
\end{abstract}

DOI: 10.1103/PhysRevLett.108.111801

PACS numbers: $13.85 . \mathrm{Rm}, 11.25 . \mathrm{Wx}, 13.85 . \mathrm{Qk}$

Over a decade ago, Arkani-Hamed, Dimopoulos, and Dvali (ADD) [1,2] proposed that extra spatial dimensions could potentially solve the standard model (SM) hierarchy problem [3], which consists of the observation of the unnatural difference of scales between the gravitational and electroweak theories. They proposed a scenario whereby the SM is constrained to the common $3+1$ space-time dimensions (brane), while gravity is free to propagate throughout a larger multidimensional space (bulk). The gravitational flux on the brane is therefore diluted by virtue of Gauss's law in the bulk, which relates the fundamental Planck scale $M_{D}$ to the apparent reduced scale $\bar{M}_{\mathrm{Pl}} \approx 2 \times 10^{18} \mathrm{GeV}$ according to the formula $M_{D}^{n_{\mathrm{ED}}+2}=\frac{\bar{M}_{\mathrm{P}}^{2}}{r_{\mathrm{ED}}}$, where $r$ and $n_{\mathrm{ED}}$ are the size and number of the extra dimensions (ED), respectively. Postulating a fundamental Planck scale to be on the order of the electroweak symmetry breaking scale $(\sim 1 \mathrm{TeV})$ results in ED with $r<1 \mathrm{~mm}$ for $n_{\mathrm{ED}} \geq 2$.

Another model of ED that solves the hierarchy problem was proposed by Randall and Sundrum (RS) [4]. In this model, the observed hierarchy is related instead to the warped anti-de Sitter (AdS) geometry of the ED. We specifically consider the RS1 model whereby only one finite ED exists separating two branes, one at each end of the ED. The geometry of the bulk is based on a slice of a five-dimensional AdS space with a length $\pi r_{c}$, where $r_{c}$ is the compactification radius. The full metric is given by $d s^{2}=e^{-k r_{\mathrm{c}} y} \eta_{\mu \nu} d x^{\mu} d x^{\nu}-r_{c}^{2} d y^{2}$, where Greek indices run over four-dimensional space-time, $\eta_{\mu \nu}$ is the

*Full author list given at the end of the article.

Published by the American Physical Society under the terms of the Creative Commons Attribution 3.0 License. Further distribution of this work must maintain attribution to the author(s) and the published article's title, journal citation, and DOI.
Minkowski metric tensor, and $0 \leq y \leq \pi$ is the coordinate along the single ED of radius $r_{c}$. The value of $k$ specifies the curvature scale (or "warp factor") and relates the fundamental Planck scale on one brane to the apparent scale on the other by $\Lambda_{\pi}=\bar{M}_{\mathrm{Pl}} e^{-k r_{c} \pi}$. As a consequence, a value of $k r_{c} \sim 10$ would provide a natural solution to the hierarchy problem, yielding $\Lambda_{\pi} \sim 1 \mathrm{TeV}$.

Phenomenologically, the excited gravitons in both models preferentially decay into two gauge bosons, such as photons, rather than into two leptons, because the graviton has spin 2, and so fermions cannot be produced in an $s$ wave. In the RS scenario, gravitons appear as wellseparated Kaluza-Klein (KK) excitations with masses and widths determined by the parameters of the RS1 model. One convenient choice of parametrization is the mass $M_{1}$ of the first excitation of the graviton and the dimensionless warp factor $\tilde{k} \equiv k / \bar{M}_{\mathrm{Pl}}$, which defines the strength of associated coupling to the SM fields. Precision electroweak data constrain $\tilde{k} \geq 0.01$, while perturbativity requirements limit $\tilde{k} \lesssim 0.10$ [5].

In the ADD model, the wave function of the KK gravitons must satisfy periodic boundary conditions, resulting in discrete energy levels with modal spacing of the order of the inverse ED size, from 1 to $100 \mathrm{meV}$, much smaller than the spacing in the RS1 model, which is expected to be of the order of $1 \mathrm{TeV}$. This effect produces an apparent continuum spectrum of diphotons, rather than distinct resonances, at high $(\sim 1 \mathrm{TeV})$ diphoton invariant mass $M_{\gamma \gamma}$.

Summing over all KK modes in the ADD scenario results in a divergence in the cross section, so an ultraviolet (UV) cutoff scale $M_{\mathrm{S}}$ is imposed. This effective Planck scale is related to-but potentially different from-the fundamental Planck scale $M_{D}$. The precise relationship depends on the UV completion of the effective theory. The effects of virtual-graviton production on the differential diphoton cross section are parametrized by the single 
variable $\eta_{G}=\mathcal{F} / M_{\mathrm{S}}^{4}$, where $\mathcal{F}$ is an order-unity dimensionless parameter, for which several conventions exist [6]. In this Letter, we set lower limits on $M_{\mathrm{S}}$ in three different conventions: GRW [7], Hewett [8], and HLZ [9].

Searches for ED via virtual-graviton effects in the ADD model have been conducted at HERA, LEP, the Tevatron, and the LHC $[10,11]$. The most stringent previously published limits on $M_{\mathrm{S}}$ for $n_{\mathrm{ED}} \geq 3$ come from the previous measurement in the diphoton channel at the Compact Muon Solenoid (CMS) experiment [6]. For $n_{\mathrm{ED}}=2$, measurements by the D0 experiment in the dijet [12] and diphoton + dielectron [13] channels are more restrictive. The most sensitive previous search for RS gravitons was conducted by the ATLAS experiment [14]. They used a search in the dilepton final state to exclude $M_{1}<1.63 \mathrm{TeV}$ for $\tilde{k}=0.10$.

In this Letter, we present a search for both nonresonant and resonant diphoton production, in the ADD and RS1 models, respectively. We use data corresponding to an integrated luminosity of $2.2 \mathrm{fb}^{-1}$, collected in $p p$ collisions at $\sqrt{s}=7 \mathrm{TeV}$ at the LHC with the CMS detector between March and August 2011.

The CMS detector [15] is designed to study collisions at the LHC. An all-silicon tracker, an electromagnetic calorimeter (ECAL), and a hadronic sampling calorimeter are all contained within a large-bore $3.8 \mathrm{~T}$ superconducting solenoid. In the central region, the tracker consists of three radial layers of silicon pixel detectors followed radially by silicon strip detectors. The finely segmented ECAL has a design resolution for unconverted photons better than $0.5 \%$ at energies exceeding $100 \mathrm{GeV}$ in the barrel $(|\eta|<1.44)$. Here, the pseudorapidity $\eta$ is defined as $-\ln [\tan (\theta / 2)]$, where $\theta$ is the polar angle with respect to the direction of the counterclockwise beam. Beyond the solenoid lie four layers of muon detectors. The instantaneous luminosity is measured with a relative uncertainty of $4.5 \%$ using information from forward hadronic calorimeters [16]. The two-tiered CMS trigger consists of the level-one trigger, composed of custom hardware, and the software-based high-level trigger.

Events for the analysis were collected through a diphoton trigger, where each photon was required to have a transverse energy $E_{T} \equiv E \sin \theta$ of at least 33 , 55, or $60 \mathrm{GeV}$, depending on the instantaneous luminosity. We require that an event be consistent with a $p p$ collision and have at least one well-reconstructed primary vertex [17]. We then reconstruct photons with $E_{T}>70 \mathrm{GeV}$ in the ECAL barrel by clustering electromagnetic energy depositions. Electrons that do not originate from photon conversions are suppressed by using information from the pixel detector to associate tracks and ECAL clusters compatible with an electron hypothesis. The probability of misidentification of an electron as a photon is approximately $3 \%$, resulting in a negligibly small contribution to the diphoton spectrum in the signal region.
Hadronic jets can be misidentified as photons when their leading hadron is an energetic $\pi^{0}$ or $\eta$ meson. We reduce the misidentification rate from this source by placing the same restrictions on the isolation as in the previous analysis for this channel [6]. These restrictions limit the total transverse energy because of tracks and calorimeter depositions near the photon cluster. Restrictions on the showershape variable $\sigma_{\eta \eta}$, which is a modified second moment of the electromagnetic energy cluster about its mean $\eta$ position [18], also suppress hadronic misidentification. Topological and timing criteria suppress anomalous signals present in the ECAL [19]. Diphoton events are selected in which $M_{\gamma \gamma}>140 \mathrm{GeV}$.

The photon reconstruction and identification efficiency is determined in Monte Carlo (MC) simulation and corrected using a data-to-MC scale factor of $1.005 \pm$ 0.034 derived from studying $Z \rightarrow e^{+} e^{-}$events. The measured efficiency for a single $E_{T}>70 \mathrm{GeV}$ photon with $|\eta|<1.44$ is $(87.4 \pm 5.4) \%$ and depends only weakly on the $E_{T}$ and $\eta$ of the photon, and the number of extra collisions present in the event. The systematic uncertainty bounds the variation as a function of these variables, the most significant of which is the number of extra collisions. We reweight the simulation to give the same reconstructed primary-vertex distribution (on average 6-8 vertices) as observed in the data. We determine the corresponding diphoton reconstruction and identification efficiency $(76.4 \pm 9.6) \%$ by squaring the single-photon efficiency.

The simulation of ED in the ADD model is performed using version 1.3.0 of the SHERPA [20] MC generator. The simulation includes both SM diphoton production and signal diphoton production via virtual-graviton exchange in order to account for the interference effects between the SM and ADD processes. The leading-order (LO) SHERPA cross sections are multiplied by a constant next-to-leadingorder (NLO) $K$ factor of $1.6 \pm 0.1$, a value that represents an updated calculation for $\sqrt{s}=7 \mathrm{TeV}$ by the authors of Refs. [21,22]. The systematic uncertainty on the signal $K$ factor reflects the approximate variation of the $K$ factor over a large region of the model parameters; it is not intended to account for the theoretical uncertainty. The cross sections in the simulation are conservatively set to zero for $\sqrt{\hat{s}}>M_{\mathrm{S}}$ because the theory becomes nonperturbative for larger values of $\sqrt{\hat{s}}$. Introducing this sharp truncation reduces the upper limits on $M_{\mathrm{S}}$ by a few percent.

The simulation of RS-graviton production is performed using version 6.424 of the PYTHIA [23] MC program. The signal cross section is scaled by a mass-dependent NLO $K$ factor [21,22], which ranges from 1.6 to 1.8 as a function of $M_{\gamma \gamma}$ and for different values of $\tilde{k}$. The CTEQ6L1 [24] parton distribution functions (PDF) are used in the simulation of both the ADD and RS models, and a $1.5 \%$ relative uncertainty on the signal acceptance is included by measuring its dependence on the choice of PDF and its uncertainties. 
Optimization of the event selection is done separately for both ADD and RS scenarios. The signal in both cases is predominantly at central values of $\eta$, while the high- $M_{\gamma \gamma}$ SM background dominates the signal in the forward region; therefore, we restrict ourselves to photons located in the ECAL barrel only. In the ADD scenario, we find that the optimal region for the search, based on the expected signal significance, is $M_{\gamma \gamma}>900 \mathrm{GeV}$. This choice of selection depends weakly on the model parameters.

In the search for RS gravitons, a fixed window is selected about the $M_{1}$ mass point of interest. Because the signal shapes deviate from Gaussian distributions, we define an effective measure of the signal width $\sigma_{\text {eff }}$ as the half-width of the narrowest mass interval containing $68 \%$ of the signal from simulation. The value of $\sigma_{\text {eff }}$ ranges from 6 to $21 \mathrm{GeV}$ for RS gravitons with $M_{1}$ between 500 and $2000 \mathrm{GeV}$ and $\tilde{k}=0.01$. The dependence on $M_{1}$ is linear and also increases with $\tilde{k}\left(\sigma_{\text {eff }}=42 \mathrm{GeV}\right.$ for $M_{1}=$ $2 \mathrm{TeV}$ and $\tilde{k}=0.10$ ). A window is then formed about the resonance mean of size $\pm 5 \sigma_{\text {eff }}$ in the data. This window contains $96 \%-97 \%$ of the signal acceptance for all mass points considered in this analysis, and the detector resolution is negligible with respect to the window size. This choice of the window maximizes the signal acceptance and analysis sensitivity in the case of small backgrounds.

Backgrounds from the misidentification of a hadronic jet as a photon are small in the signal region but contribute to the low- $M_{\gamma \gamma}$ region. Two such sources of backgrounds from isolated-photon misidentification are considered: multijet production and prompt single-photon $(\gamma+$ jet $)$ production. In particular, we measure on a backgrounddominated sample a misidentification rate, defined as the ratio of the number of isolated photon candidates to nonisolated photonlike objects. These photonlike objects are reconstructed as photons but fail one of the isolation or shower-shape criteria; therefore, the samples corresponding to numerator and denominator are mutually exclusive, and prompt photons have a negligibly small contribution to the denominator. The misidentification rate is measured in a photon-triggered sample in bins of photon(like) candidate $E_{T}$, but the objects used in the measurement are required to be well separated from the triggered object to avoid a trigger-induced bias.

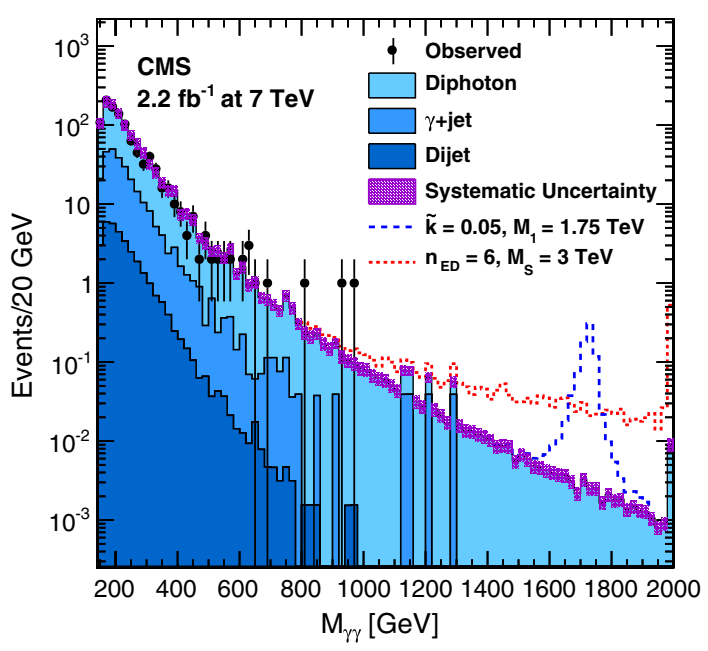

FIG. 1 (color online). Observed event yields (points with error bars) and background expectations (filled solid histograms) as a function of the diphoton invariant mass. Photons are required to be isolated, with $E_{T}>70 \mathrm{GeV}$ and $|\eta|<1.44$. The shaded band around the background estimation corresponds to the average systematic uncertainty over the spectrum. The precise per-bin uncertainty is not provided for the sake of clarity. The last bin includes the sum of all contributions for $M_{\gamma \gamma}>2.0 \mathrm{TeV}$. The simulated distributions for two, nonexcluded signal hypotheses are shown for comparison as dotted (ADD) and dashed (RS) lines.

Because the background-dominated sample in which we measure the misidentification rate may contain some genuine, isolated photons that "contaminate" the numerator of the misidentification rate, we correct for this on a bin-bybin basis. The $\sigma_{\eta \eta}$ requirement is released and the numerator sample is fit for the fraction of prompt photons using one-dimensional probability density histograms ("templates") in $\sigma_{\eta \eta}$. The signal template is constructed from MC simulation, and the background template is constructed from reconstructed photons that fail one or more of the isolation criteria. The measured misidentification rate falls from $7 \%$ at $E_{T}=70 \mathrm{GeV}$ to $2 \%$ at $E_{T}=$ $120 \mathrm{GeV}$. We apply a $20 \%$ systematic uncertainty to the rate derived from the variation of the misidentification rate measured in a jet-triggered sample.

TABLE I. Observed event yields and background expectations for different reconstructed diphoton invariant-mass ranges. Full systematic uncertainties are included.

\begin{tabular}{lcccc}
\hline \hline & & \multicolumn{3}{c}{ Diphoton invariant-mass range $[\mathrm{TeV}]$} \\
Process & {$[0.14,0.2]$} & {$[0.2,0.5]$} & {$[0.5,0.9]$} & $>0.9$ \\
\hline Multijet & $15 \pm 6$ & $17 \pm 7$ & $0.2 \pm 0.1$ & $0.003 \pm 0.001$ \\
$\gamma+$ jet & $102 \pm 15$ & $124 \pm 18$ & $2.5 \pm 0.4$ & $0.19 \pm 0.04$ \\
Diphoton & $372 \pm 70$ & $414 \pm 78$ & $16.9 \pm 3.2$ & $1.3 \pm 0.3$ \\
Backgrounds & $489 \pm 73$ & $555 \pm 81$ & $19.6 \pm 3.2$ & $1.5 \pm 0.3$ \\
Observed & 484 & 517 & 16 & 2 \\
\hline \hline
\end{tabular}


The multijet and $\gamma+$ jet backgrounds to the reconstructed diphoton spectrum are estimated by using the misidentification rate to extrapolate from two background-dominated reference regions, both selected with the same diphoton trigger as the primary signal sample. One region includes events with only one isolated photon, but one or more nonisolated photons. The other region includes events with no isolated photons, but two or more nonisolated photons. The diphoton trigger is sufficiently inclusive that the regions are unaffected by the trigger selection. By applying the prompt-photon misidentification rate to these two reference regions, we predict the $\gamma+$ jet and multijet backgrounds in the signal region.

The SM diphoton background dominates the signal region. The expected number of background events due to this process is computed by rescaling the prediction from PYTHIA with a NLO $K$ factor that varies with $M_{\gamma \gamma}$. The NLO prediction is calculated with the DIPHOX+ GAMMA2MC $[25,26]$ generators, which take into account the fragmentation processes in which the photons can come from the collinear fragmentations of hard partons. A separate analysis by CMS has also demonstrated good agreement with the NLO prediction at low $M_{\gamma \gamma} \lesssim$ $300 \mathrm{GeV}$ [27]. The subleading-order gluon-fusion box diagram is included as a part of the PYTHIA calculation because of its large contribution at the LHC energy, although its effects are small at high $M_{\gamma \gamma}$. The $K$ factor varies between 1.7 and 1.1 from low to high $M_{\gamma \gamma}$. A systematic uncertainty of $15 \%$ on the value of the $K$ factor is determined by examining the PDF uncertainties and variation of the renormalization and factorization scales.

Figure 1 shows the invariant-mass distribution of the selected events, together with the estimated distributions for each of the backgrounds. Table I presents the observed number of events in the data and the predicted number of background events in different ranges in $M_{\gamma \gamma}$ and corresponds directly to Fig. 1. The last column corresponds to the signal region for the ADD search. We find that the observed data are consistent with the background estimate throughout the $M_{\gamma \gamma}$ spectrum and do not show an excess of events, neither resonant nor nonresonant.
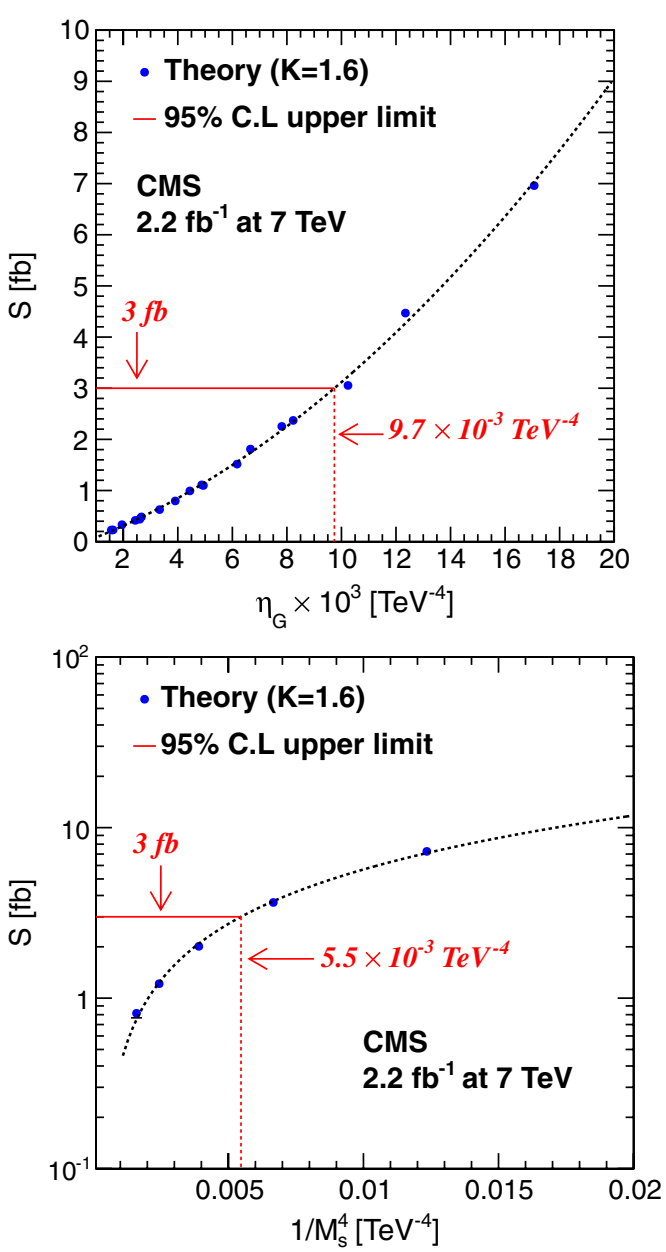

FIG. 2 (color online). Signal cross section $S$ parametrization as a function of the strength of the ED effects $\eta_{G}$ (top) and as a function of $1 / M_{\mathrm{S}}^{4}$ for the $\mathrm{HLZ} n_{\mathrm{ED}}=2$ case (bottom).

To set limits on virtual-graviton exchange in the ADD scenario, we compare the number of observed and expected events in the signal region $\left(M_{\gamma \gamma}>0.9 \mathrm{TeV}\right)$ and set $95 \%$ confidence level (C.L.) upper limits on the quantity $S \equiv\left(\sigma_{\text {total }}-\sigma_{\mathrm{SM}}\right) \times \mathcal{B} \times \mathcal{A}$, where $\sigma_{\text {total }}$ represents the total diphoton production cross section (including signal, SM, and interference effects), and $\sigma_{\mathrm{SM}}$ represents

TABLE II. The 95\% C.L. lower limits on $M_{\mathrm{S}}$ (in TeV) in the GRW, Hewett, and HLZ conventions for two values of the ADD signal $K$ factor, 1.0 and $1.6 \pm 0.1$. All limits are computed with a signal cross section truncated to zero for $\sqrt{\hat{s}}>M_{\mathrm{S}}$, where $\sqrt{\hat{s}}$ is the centerof-mass of the partonic collision. The limits are presented for both positive and negative interference in the Hewett convention and for $n_{\mathrm{ED}}=2-7$ in the HLZ convention. The median expected lower limits are given in parentheses.

\begin{tabular}{lccccccccc}
\hline \hline \multicolumn{1}{c}{ Hewett } & \multicolumn{9}{c}{ HLZ } \\
$K$ & GRW & Positive & Negative & $n_{\mathrm{ED}}=2$ & $n_{\mathrm{ED}}=3$ & $n_{\mathrm{ED}}=4$ & $n_{\mathrm{ED}}=5$ & $n_{\mathrm{ED}}=6$ & $n_{\mathrm{ED}}=7$ \\
\hline 1.0 & 2.94 & 2.63 & 2.28 & 3.29 & 3.50 & 2.94 & 2.66 & 2.47 & 2.34 \\
& $(2.99)$ & $(2.67)$ & $(2.31)$ & $(3.37)$ & $(3.56)$ & $(2.99)$ & $(2.71)$ & $(2.52)$ & $(2.38)$ \\
$1.6 \pm 0.1$ & 3.18 & 2.84 & 2.41 & 3.68 & 3.79 & 3.18 & 2.88 & 2.68 & 2.53 \\
& $(3.24)$ & $(2.90)$ & $(2.44)$ & $(3.77)$ & $(3.85)$ & $(3.24)$ & $(2.93)$ & $(2.73)$ & $(2.58)$ \\
\hline \hline
\end{tabular}




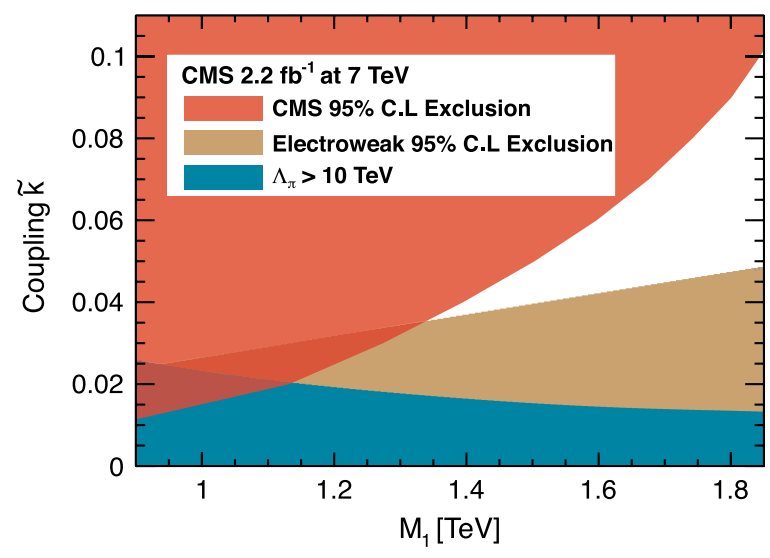

FIG. 3 (color online). The 95\% C.L. exclusion region for the RS1 graviton model in the $M_{1}-\tilde{k}$ plane. The expected limits coincide very closely with the measured limits and so are not shown in the figure. Also shown are bounds due to electroweak constraints and naturalness $\left(\Lambda_{\pi}>10 \mathrm{TeV}\right)$. Perturbativity requirements bound $\tilde{k} \lesssim 0.10$.

the SM diphoton production cross section. The signal branching fraction to diphotons is indicated by $\mathcal{B}$ and the signal acceptance by $\mathcal{A}$. We use the $\mathrm{CL}_{s}$ technique $[28,29]$ to compute the limits with a likelihood constructed from the Poisson probability to observe $N$ events, given $S$, the signal efficiency $(76.4 \pm 9.6) \%$, the expected number of background events $(1.5 \pm 0.3)$, and the integrated luminosity $\mathcal{L}=(2.2 \pm 0.1) \mathrm{fb}^{-1}$ [16]. The variation of the $K$ factor is included in the statistical analysis as an uncertainty on the signal yield.

The observed (median expected) 95\% C.L. upper limit on $S$ is $3.0 \mathrm{fb}(2.7 \mathrm{fb})$. For the HLZ $n_{\mathrm{ED}}=2$ case, we parametrize $S$ directly as a smooth function of $1 / M_{\mathrm{S}}^{4}$. For all other conventions, $S$ is parametrized as a function of the parameter $\eta_{G}$, as in Ref. [6]. The observed 95\% C.L. limit, together with the signal parametrization, is shown in Fig. 2. The intersection of the cross-section limit with the parametrized curve determines the 95\% C.L. upper limit on the parameter $\eta_{G}$. As seen from the plot, these upper limits on $S$ correspond to upper limits of $\eta_{G} \leq$ $0.0097 \mathrm{TeV}^{-4}$ and $1 / M_{\mathrm{S}}^{4} \leq 0.0055 \mathrm{TeV}^{-4}$. The upper limits on $\eta_{G}$ are equated to lower limits on $M_{\mathrm{S}}$ and are shown in Table II.

For the RS scenario, the same limit-setting calculation is performed, but in a bounded window in $M_{\gamma \gamma}$. Figure 3 shows the excluded regions in the $M_{1}-\tilde{k}$ plane. Also shown are bounds due to precision electroweak measurements and to naturalness arguments [5]. Table III presents the $95 \%$ C.L. lower limits on the graviton mass $M_{1}$ for different values of $\tilde{k}$. The median expected lower limits coincide within a few $\mathrm{GeV}$ of the observed limits.

In summary, we have performed a search for extra spatial dimensions leading to enhanced resonant or nonresonant diphoton production in proton-proton collisions at a center-of-mass energy of $7 \mathrm{TeV}$ at the LHC. Using a data sample corresponding to an integrated luminosity of $2.2 \mathrm{fb}^{-1}$ recorded by the CMS experiment, we observe no excess in diphoton production above the rate predicted from SM background sources. Values of the effective Planck scale $M_{\mathrm{S}}$ less than $2.3-3.8 \mathrm{TeV}$ are excluded at $95 \%$ C.L. for ADD models. We also exclude at $95 \%$ C.L. resonant graviton production in the RS1 model with values of $M_{1}$ less than 0.86-1.84 TeV depending on the normalized coupling strength $\tilde{k}$. We present limits on both the ADD and RS1 models of extra dimensions in the diphoton final state that extend those observed at the D0 experiment [13], as well as those set previously by the CMS [6] and ATLAS [14] experiments.

We thank M. C. Kumar, P. Mathews, V. Ravindran, and A. Tripathi for the calculation of NLO $K$ factors used in this Letter. We wish to congratulate our colleagues in the CERN accelerator departments for the excellent performance of the LHC machine. We thank the technical and administrative staff at CERN and other CMS institutes, and acknowledge support from FMSR (Austria); FNRS and FWO (Belgium); CNPq, CAPES, FAPERJ, and FAPESP (Brazil); MES (Bulgaria); CERN; CAS, MoST, and NSFC (China); COLCIENCIAS (Colombia); MSES (Croatia); RPF (Cyprus); Academy of Sciences and NICPB (Estonia); Academy of Finland, MEC, and HIP (Finland); CEA and CNRS/IN2P3 (France); BMBF, DFG, and HGF (Germany); GSRT (Greece); OTKA and NKTH (Hungary); DAE and DST (India); IPM (Iran); SFI (Ireland); INFN (Italy); NRF and WCU (Korea); LAS (Lithuania); CINVESTAV, CONACYT, SEP, and UASLP-FAI (Mexico); MSI (New Zealand); PAEC (Pakistan); SCSR (Poland); FCT (Portugal); JINR (Armenia, Belarus, Georgia, Ukraine, Uzbekistan); MST, MAE, and RFBR (Russia); MSTD (Serbia); MICINN and CPAN (Spain); Swiss Funding Agencies (Switzerland); NSC (Taipei); TUBITAK and TAEK (Turkey); STFC (United Kingdom); DOE and NSF (U.S.).

TABLE III. The $95 \%$ C.L. lower limits on $M_{1}$ for given values of the coupling parameter $\tilde{k}$. For $\tilde{k}<0.03$, masses above the presented limits are excluded by electroweak and naturalness constraints. The median expected lower limits are numerically the same for the presented precision except for the $\tilde{k}=0.01$ case, for which the expected lower limit on $M_{1}$ is $0.84 \mathrm{TeV}$.

\begin{tabular}{llllllllll}
\hline \hline$\tilde{k}$ & 0.01 & 0.02 & 0.03 & 0.04 & 0.05 & 0.06 & 0.07 & 0.08 & 0.09 \\
$M_{1}[\mathrm{TeV}]$ & 0.86 & 1.13 & 1.27 & 1.39 & 1.50 & 1.59 & 1.67 & 1.74 & 1.80 \\
\hline \hline
\end{tabular}


[1] N. Arkani-Hamed, S. Dimopoulos, and G. Dvali, Phys. Lett. B 429, 263 (1998).

[2] N. Arkani-Hamed, S. Dimopoulos, and G. Dvali, Phys. Rev. D 59, 086004 (1999).

[3] E. Witten, Phys. Lett. B 105, 267 (1981).

[4] L. Randall and R. Sundrum, Phys. Rev. Lett. 83, 3370 (1999).

[5] H. Davoudiasl, J. L. Hewett, and T. G. Rizzo, Phys. Rev. D 63, 075004 (2001).

[6] S. Chatrchyan et al. (CMS Collaboration), J. High Energy Phys. 05 (2011) 85.

[7] G. Giudice, R. Rattazzi, and J. Wells, Nucl. Phys. B544, 3 (1999).

[8] J. L. Hewett, Phys. Rev. Lett. 82, 4765 (1999).

[9] T. Han, J. D. Lykken, and R.-J. Zhang, Phys. Rev. D 59, 105006 (1999).

[10] K. Nakamura et al. (Particle Data Group), J. Phys. G 37, 075021 (2010).

[11] K. Cheung and G. Landsberg, Phys. Rev. D 62, 076003 (2000).

[12] V. M. Abazov et al. (D0 Collaboration), Phys. Rev. Lett. 103, 191803 (2009).

[13] V. M. Abazov et al. (D0 Collaboration), Phys. Rev. Lett. 102, 051601 (2009).

[14] G. Aad et al. (ATLAS Collaboration), Phys. Rev. Lett. 107, 272002 (2011).

[15] S. Chatrchyan et al. (CMS Collaboration), JINST 3, S08004 (2008).
[16] CMS Collaboration, Report No. CMS-PAS-EWK-10-004, 2010 [https://cdsweb.cern.ch/record/1279145?ln=en].

[17] V. Khachatryan et al. (CMS Collaboration), Eur. Phys. J. C 70, 1165 (2010).

[18] V. Khachatryan et al. (CMS Collaboration), Phys. Rev. Lett. 106, 082001 (2011).

[19] CMS Collaboration, Report No. CMS-PAS-EGM-10-006, 2010 [https://cdsweb.cern.ch/record/1324545?ln=en].

[20] T. Gleisberg et al., J. High Energy Phys. 02 (2004) 056.

[21] M. Kumar, P. Mathews, V. Ravindran, and A. Tripathi, Phys. Lett. B 672, 45 (2009).

[22] M. Kumar, P. Mathews, V. Ravindran, and A. Tripathi, Nucl. Phys. B818, 28 (2009).

[23] T. Sjostrand, P. Eden, C. Friberg, L. Lonnblad, G. Miu, S. Mrenna, and E. Norrbin, Comput. Phys. Commun. 135, 238 (2001).

[24] P. Nadolsky et al. (CTEQ Collaboration), Phys. Rev. D 78, 013004 (2008).

[25] T. Binoth, J. P. Guillet, E. Pilon, and M. Werlen, Eur. Phys. J. C 16, 311 (2000).

[26] Z. Bern, L. Dixon, and C. Schmidt, Phys. Rev. D 66, 074018 (2002).

[27] S. Chatrchyan et al. (CMS Collaboration), J. High Energy Phys. 01 (2012) 133.

[28] A. L. Read, J. Phys. G 28, 2693 (2002).

[29] T. Junk, Nucl. Instrum. Methods Phys. Res., Sect. A 434, 435 (1999).

S. Chatrchyan, ${ }^{1}$ V. Khachatryan, ${ }^{1}$ A. M. Sirunyan, ${ }^{1}$ A. Tumasyan, ${ }^{1}$ W. Adam, ${ }^{2}$ T. Bergauer, ${ }^{2}$ M. Dragicevic, ${ }^{2}$ J. Erö, ${ }^{2}$ C. Fabjan, ${ }^{2}$ M. Friedl, ${ }^{2}$ R. Frühwirth, ${ }^{2}$ V. M. Ghete, ${ }^{2}$ J. Hammer, ${ }^{2, b}$ M. Hoch, ${ }^{2}$ N. Hörmann, ${ }^{2}$ J. Hrubec, ${ }^{2}$ M. Jeitler, ${ }^{2}$ W. Kiesenhofer, ${ }^{2}$ A. Knapitsch, ${ }^{2}$ M. Krammer, ${ }^{2}$ D. Liko, ${ }^{2}$ I. Mikulec, ${ }^{2}$ M. Pernicka, ${ }^{2, a}$ B. Rahbaran, ${ }^{2}$ H. Rohringer, ${ }^{2}$ R. Schöfbeck, ${ }^{2}$ J. Strauss, ${ }^{2}$ A. Taurok, ${ }^{2}$ F. Teischinger, ${ }^{2}$ C. Trauner, ${ }^{2}$ P. Wagner, ${ }^{2}$ W. Waltenberger, ${ }^{2}$ G. Walzel, ${ }^{2}$ E. Widl, ${ }^{2}$ C.-E. Wulz, ${ }^{2}$ V. Mossolov, ${ }^{3}$ N. Shumeiko, ${ }^{3}$ J. Suarez Gonzalez, ${ }^{3}$ S. Bansal, ${ }^{4}$ L. Benucci, ${ }^{4}$ E. A. De Wolf, ${ }^{4}$ X. Janssen, ${ }^{4}$ S. Luyckx, ${ }^{4}$ T. Maes, ${ }^{4}$ L. Mucibello, ${ }^{4}$ S. Ochesanu, ${ }^{4}$ B. Roland, ${ }^{4}$ R. Rougny, ${ }^{4}$ M. Selvaggi, ${ }^{4}$ H. Van Haevermaet, ${ }^{4}$ P. Van Mechelen, ${ }^{4}$ N. Van Remortel, ${ }^{4}$ A. Van Spilbeeck, ${ }^{4}$ F. Blekman, ${ }^{5}$ S. Blyweert, ${ }^{5}$ J. D'Hondt, ${ }^{5}$ R. Gonzalez Suarez, ${ }^{5}$ A. Kalogeropoulos, ${ }^{5}$ M. Maes, ${ }^{5}$ A. Olbrechts, ${ }^{5}$ W. Van Doninck, ${ }^{5}$ P. Van Mulders, ${ }^{5}$ G. P. Van Onsem, ${ }^{5}$ I. Villella,${ }^{5}$ O. Charaf, ${ }_{6}^{6}$ B. Clerbaux ${ }^{6}{ }^{6}$ G. De Lentdecker, ${ }^{6}$ V. Dero, ${ }^{6}$ A. P. R. Gay, ${ }^{6}$ G. H. Hammad, ${ }^{6}$ T. Hreus, ${ }^{6}$ A. Léonard, ${ }^{6}$ P. E. Marage, ${ }^{6}$ L. Thomas, ${ }^{6}$ C. Vander Velde, ${ }^{6}$ P. Vanlaer, ${ }^{6}$ J. Wickens, ${ }^{6}$

V. Adler, ${ }^{7}$ K. Beernaert, ${ }^{7}$ A. Cimmino, ${ }^{7}$ S. Costantini, ${ }^{7}$ M. Grunewald, ${ }^{7}$ B. Klein, ${ }^{7}$ J. Lellouch, ${ }^{7}$ A. Marinov, ${ }^{7}$

J. Mccartin, ${ }^{7}$ D. Ryckbosch ${ }^{7}$ N. Strobbe,${ }^{7}$ F. Thyssen, ${ }^{7}$ M. Tytgat, ${ }^{7}$ L. Vanelderen, ${ }^{7}$ P. Verwilligen, ${ }^{7}$ S. Walsh, ${ }^{7}$ N. Zaganidis, ${ }^{7}$ S. Basegmez, ${ }^{8}$ G. Bruno, ${ }^{8}$ J. Caudron, ${ }^{8}$ L. Ceard, ${ }^{8}$ J. De Favereau De Jeneret, ${ }^{8}$ C. Delaere, ${ }^{8}$ D. Favart, ${ }^{8}$

L. Forthomme, ${ }^{8}$ A. Giammanco, ${ }^{8, \mathrm{c}}$ G. Grégoire, ${ }^{8}$ J. Hollar, ${ }^{8}$ V. Lemaitre, ${ }^{8}$ J. Liao, ${ }^{8}$ O. Militaru, ${ }^{8}$ C. Nuttens, ${ }^{8}$

D. Pagano, ${ }^{8}$ A. Pin, ${ }^{8}$ K. Piotrzkowski, ${ }^{8}$ N. Schul,${ }^{8}$ N. Beliy, ${ }^{9}$ T. Caebergs, ${ }^{9}$ E. Daubie, ${ }^{9}$ G. A. Alves, ${ }^{10}$ D. De Jesus Damiao, ${ }^{10}$ M. E. Pol, ${ }^{10}$ M. H. G. Souza, ${ }^{10}$ W. L. Aldá Júnior, ${ }^{11}$ W. Carvalho, ${ }^{11}$ A. Custódio,,${ }^{11}$ E. M. Da Costa, ${ }^{11}$ C. De Oliveira Martins, ${ }^{11}$ S. Fonseca De Souza, ${ }^{11}$ D. Matos Figueiredo, ${ }^{11}$ L. Mundim,,${ }^{11}$ H. Nogima, ${ }^{11}$ V. Oguri, ${ }^{11}$ W. L. Prado Da Silva, ${ }^{11}$ A. Santoro, ${ }^{11}$ S. M. Silva Do Amaral, ${ }^{11}$ A. Sznajder,${ }^{11}$ T. S. Anjos, ${ }^{12, \mathrm{~d}}$ C. A. Bernardes, ${ }^{12, \mathrm{~d}}$ F. A. Dias, ${ }^{12, \mathrm{e}}$ T. R. Fernandez Perez Tomei, ${ }^{12}$ E. M. Gregores, ${ }^{12, \mathrm{~d}}$ C. Lagana, ${ }^{12}$ F. Marinho, ${ }^{12}$ P. G. Mercadante, ${ }^{12, \mathrm{~d}}$ S. F. Novaes, ${ }^{12}$ Sandra S. Padula, ${ }^{12}$ N. Darmenov, ${ }^{13, \mathrm{~b}}$ V. Genchev, ${ }^{13, \mathrm{~b}}$ P. Iaydjiev, ${ }^{13, b}$ S. Piperov, ${ }^{13}$ M. Rodozov, ${ }^{13}$ S. Stoykova, ${ }^{13}$ G. Sultanov, ${ }^{13}$ V. Tcholakov, ${ }^{13}$ R. Trayanov, ${ }^{13}$ M. Vutova, ${ }^{13}$ A. Dimitrov, ${ }^{14}$ R. Hadjiiska, ${ }^{14}$ A. Karadzhinova, ${ }^{14}$ V. Kozhuharov,${ }^{14}$ L. Litov ${ }^{14}$ B. Pavlov, ${ }^{14}$ P. Petkov, ${ }^{14}$ J. G. Bian, ${ }^{15}$ G. M. Chen, ${ }^{15}$ H. S. Chen, ${ }^{15}$ C. H. Jiang,${ }^{15}$ D. Liang, ${ }^{15}$ S. Liang, ${ }^{15}$ X. Meng, ${ }^{15}$ J. Tao, ${ }^{15}$ J. Wang, ${ }^{15}$ J. Wang, ${ }^{15}$ X. Wang, ${ }^{15}$ Z. Wang, ${ }^{15}$ H. Xiao, ${ }^{15}$ M. Xu, ${ }^{15}$ J. Zang, ${ }^{15}$ Z. Zhang, ${ }^{15}$ Y. Ban, ${ }^{16}$ S. Guo, ${ }^{16}$ Y. Guo, ${ }^{16}$ W. Li, ${ }^{16}$ S. Liu, ${ }^{16}$ Y. Mao, ${ }^{16}$ S. J. Qian, ${ }^{16}$ H. Teng, ${ }^{16}$ S. Wang, ${ }^{16}$ B. Zhu, ${ }^{16}$ W. Zou, ${ }^{16}$ A. Cabrera, ${ }^{17}$ 
B. Gomez Moreno, ${ }^{17}$ A. A. Ocampo Rios,${ }^{17}$ A. F. Osorio Oliveros,${ }^{17}$ J. C. Sanabria, ${ }^{17}$ N. Godinovic,${ }^{18}$ D. Lelas,${ }^{18}$ R. Plestina,${ }^{18, \mathrm{f}}$ D. Polic, ${ }^{18}$ I. Puljak, ${ }^{18, \mathrm{~b}}$ Z. Antunovic, ${ }^{19}$ M. Dzelalija, ${ }^{19} \mathrm{M}$. Kovac, ${ }^{19}$ V. Brigljevic,${ }^{20}$ S. Duric,${ }^{20}$ K. Kadija ${ }^{20}$ J. Luetic,${ }^{20}$ S. Morovic,${ }^{20}$ A. Attikis, ${ }^{21}$ M. Galanti,${ }^{21}$ J. Mousa,${ }^{21}$ C. Nicolaou, ${ }^{21}$ F. Ptochos,${ }^{21}$ P. A. Razis, ${ }^{21}$ M. Finger, ${ }^{22}$ M. Finger, Jr., ${ }^{22}$ Y. Assran, ${ }^{23, g}$ A. Ellithi Kamel, ${ }^{23, h}$ S. Khalil,${ }^{23, i}$ M. A. Mahmoud ${ }^{23, j}$ A. Radi, ${ }^{23, \mathrm{k}}$ A. Hektor, ${ }^{24}$ M. Kadastik,,${ }^{24}$ M. Müntel, ${ }^{24}$ M. Raidal, ${ }^{24}$ L. Rebane,${ }^{24}$ A. Tiko, ${ }^{24}$ V. Azzolini, ${ }^{25}$ P. Eerola, ${ }^{25}$

G. Fedi, ${ }^{25}$ M. Voutilainen, ${ }^{25}$ S. Czellar, ${ }^{26}$ J. Härkönen, ${ }^{26}$ A. Heikkinen, ${ }^{26}$ V. Karimäki, ${ }^{26}$ R. Kinnunen, ${ }^{26}$

M. J. Kortelainen, ${ }^{26}$ T. Lampén, ${ }^{26} \mathrm{~K}$. Lassila-Perini, ${ }^{26} \mathrm{~S}$. Lehti, ${ }^{26} \mathrm{~T}$. Lindén, ${ }^{26} \mathrm{P}$. Luukka, ${ }^{26} \mathrm{~T}$. Mäenpää, ${ }^{26}$

E. Tuominen ${ }^{26}$ J. Tuominiemi, ${ }^{26}$ E. Tuovinen, ${ }^{26}$ D. Ungaro, ${ }^{26}$ L. Wendland,${ }^{26} \mathrm{~K}$. Banzuzi, ${ }^{27}$ A. Karjalainen,${ }^{27}$ A. Korpela, ${ }^{27}$ T. Tuuva, ${ }^{27}$ D. Sillou, ${ }^{28}$ M. Besancon ${ }^{29}$ S. Choudhury, ${ }^{29}$ M. Dejardin, ${ }^{29}$ D. Denegri, ${ }^{29}$ B. Fabbro, ${ }^{29}$ J. L. Faure ${ }^{29}$ F. Ferri, ${ }^{29}$ S. Ganjour, ${ }^{29}$ A. Givernaud, ${ }^{29}$ P. Gras,${ }^{29}$ G. Hamel de Monchenault, ${ }^{29}$ P. Jarry, ${ }^{29}$ E. Locci, ${ }^{29}$ J. Malcles, ${ }^{29}$ M. Marionneau, ${ }^{29}$ L. Millischer ${ }^{29}$ J. Rander, ${ }^{29}$ A. Rosowsky,${ }^{29}$ I. Shreyber,${ }^{29}$ M. Titov, ${ }^{29}$ S. Baffioni, ${ }^{30}$

F. Beaudette, ${ }^{30}$ L. Benhabib, ${ }^{30}$ L. Bianchini, ${ }^{30}$ M. Bluj, ${ }^{30,1}$ C. Broutin, ${ }^{30}$ P. Busson, ${ }^{30}$ C. Charlot,${ }^{30}$ N. Daci, ${ }^{30}$ T. Dahms,${ }^{30}$ L. Dobrzynski, ${ }^{30}$ S. Elgammal,${ }^{30}$ R. Granier de Cassagnac,${ }^{30}$ M. Haguenauer, ${ }^{30}$ P. Miné, ${ }^{30}$ C. Mironov, ${ }^{30}$ C. Ochando,${ }^{30}$ P. Paganini, ${ }^{30}$ D. Sabes,${ }^{30}$ R. Salerno, ${ }^{30}$ Y. Sirois,${ }^{30}$ C. Thiebaux,${ }^{30}$ C. Veelken, ${ }^{30}$ A. Zabi, ${ }^{30}$ J.-L. Agram,${ }^{31, \mathrm{~m}}$ J. Andrea, ${ }^{31}$ D. Bloch, ${ }^{31}$ D. Bodin, ${ }^{31}$ J.-M. Brom,${ }^{31}$ M. Cardaci, ${ }^{31}$ E. C. Chabert, ${ }^{31}$ C. Collard, ${ }^{31}$ E. Conte, ${ }^{31, \mathrm{~m}}$ F. Drouhin, ${ }^{31, \mathrm{~m}}$ C. Ferro, ${ }^{31}$ J.-C. Fontaine, ${ }^{31, \mathrm{~m}}$ D. Gelé, ${ }^{31}$ U. Goerlach, ${ }^{31}$ S. Greder, ${ }^{31}$ P. Juillot, ${ }^{31}$ M. Karim, ${ }^{31, \mathrm{~m}}$ A.-C. Le Bihan, ${ }^{31}$ P. Van Hove, ${ }^{31}$ F. Fassi, ${ }^{32}$ D. Mercier, ${ }^{32}$ C. Baty, ${ }^{33}$ S. Beauceron, ${ }^{33}$ N. Beaupere, ${ }^{33}$ M. Bedjidian, ${ }^{33}$ O. Bondu, ${ }^{33}$ G. Boudoul, ${ }^{33}$ D. Boumediene, ${ }^{33}$ H. Brun, ${ }^{33}$ J. Chasserat,${ }^{33}$ R. Chierici, ${ }^{33, b}$

D. Contardo, ${ }^{33}$ P. Depasse, ${ }^{33}$ H. El Mamouni, ${ }^{33}$ A. Falkiewicz, ${ }^{33}$ J. Fay, ${ }^{33}$ S. Gascon, ${ }^{33}$ M. Gouzevitch,${ }^{33}$ B. Ille, ${ }^{33}$

T. Kurca, ${ }^{33}$ T. Le Grand, ${ }^{33}$ M. Lethuillier, ${ }^{33}$ L. Mirabito, ${ }^{33}$ S. Perries, ${ }^{33}$ V. Sordini, ${ }^{33}$ S. Tosi, ${ }^{33}$ Y. Tschudi, ${ }^{33}$ P. Verdier, ${ }^{33}$ S. Viret ${ }^{33}$ D. Lomidze,${ }^{34}$ G. Anagnostou, ${ }^{35}$ S. Beranek,${ }^{35}$ M. Edelhoff, ${ }^{35}$ L. Feld,${ }^{35}$ N. Heracleous,${ }^{35}$ O. Hindrichs, ${ }^{35}$ R. Jussen, ${ }^{35}$ K. Klein, ${ }^{35}$ J. Merz,${ }^{35}$ A. Ostapchuk,,${ }^{35}$ A. Perieanu, ${ }^{35}$ F. Raupach, ${ }^{35}$ J. Sammet,${ }^{35}$ S. Schael,${ }^{35}$ D. Sprenger, ${ }^{35}$ H. Weber, ${ }^{35}$ B. Wittmer, ${ }^{35}$ V. Zhukov, ${ }^{35, n}$ M. Ata,${ }^{36}$ E. Dietz-Laursonn, ${ }^{36}$ M. Erdmann, ${ }^{36}$ T. Hebbeker, ${ }^{36}$ C. Heidemann, ${ }^{36}$ A. Hinzmann, ${ }^{36}$ K. Hoepfner, ${ }^{36}$ T. Klimkovich, ${ }^{36}$ D. Klingebiel, ${ }^{36}$ P. Kreuzer, ${ }^{36}$ D. Lanske, ${ }^{36, a}$ J. Lingemann, ${ }^{36}$ C. Magass, ${ }^{36}$ M. Merschmeyer, ${ }^{36}$ A. Meyer, ${ }^{36}$ P. Papacz, ${ }^{36}$ H. Pieta, ${ }^{36}$ H. Reithler,${ }^{36}$ S. A. Schmitz, ${ }^{36}$ L. Sonnenschein, ${ }^{36}$ J. Steggemann, ${ }^{36}$ D. Teyssier, ${ }^{36}$ M. Weber, ${ }^{36}$ M. Bontenackels, ${ }^{37}$ V. Cherepanov, ${ }^{37}$ M. Davids, ${ }^{37}$ G. Flügge, ${ }^{37}$ H. Geenen, ${ }^{37}$ W. Haj Ahmad, ${ }^{37}$ F. Hoehle, ${ }^{37}$ B. Kargoll, ${ }^{37}$ T. Kress, ${ }^{37}$ Y. Kuessel,,${ }^{37}$ A. Linn, ${ }^{37}$ A. Nowack, ${ }^{37}$ L. Perchalla, ${ }^{37}$ O. Pooth, ${ }^{37}$ J. Rennefeld, ${ }^{37}$ P. Sauerland, ${ }^{37}$ A. Stahl,,${ }^{37}$ D. Tornier, ${ }^{37}$ M. H. Zoeller,${ }^{37}$ M. Aldaya Martin, ${ }^{38}$ W. Behrenhoff, ${ }^{38}$ U. Behrens,${ }^{38}$ M. Bergholz,${ }^{38, o}$ A. Bethani, ${ }^{38}$ K. Borras, ${ }^{38}$ A. Cakir, ${ }^{38}$ A. Campbell,,${ }^{38}$ E. Castro, ${ }^{38}$ D. Dammann, ${ }^{38}$ G. Eckerlin, ${ }^{38}$ D. Eckstein,${ }^{38}$ A. Flossdorf, ${ }^{38}$ G. Flucke, ${ }^{38}$ A. Geiser,${ }^{38}$ J. Hauk,${ }^{38}$ H. Jung, ${ }^{38, b}$ M. Kasemann, ${ }^{38}$ P. Katsas, ${ }^{38}$ C. Kleinwort, ${ }^{38}$ H. Kluge, ${ }^{38}$ A. Knutsson, ${ }^{38}$ M. Krämer, ${ }^{38}$ D. Krücker, ${ }^{38}$ E. Kuznetsova, ${ }^{38}$ W. Lange,${ }^{38}$ W. Lohmann, ${ }^{38, o}$ B. Lutz, ${ }^{38}$ R. Mankel, ${ }^{38}$ I. Marfin, ${ }^{38}$ M. Marienfeld, ${ }^{38}$ I.-A. Melzer-Pellmann, ${ }^{38}$ A. B. Meyer, ${ }^{38}$ J. Mnich, ${ }^{38}$ A. Mussgiller, ${ }^{38}$ S. Naumann-Emme, ${ }^{38}$ J. Olzem, ${ }^{38}$ A. Petrukhin, ${ }^{38}$ D. Pitzl, ${ }^{38}$ A. Raspereza,${ }^{38}$ M. Rosin, ${ }^{38}$ J. Salfeld-Nebgen, ${ }^{38}$ R. Schmidt, ${ }^{38,0}$ T. Schoerner-Sadenius, ${ }^{38}$ N. Sen, ${ }^{38}$ A. Spiridonov, ${ }^{38}$ M. Stein, ${ }^{38}$ J. Tomaszewska, ${ }^{38}$ R. Walsh, ${ }^{38}$ C. Wissing, ${ }^{38}$ C. Autermann, ${ }^{39}$ V. Blobel, ${ }^{39}$ S. Bobrovskyi, ${ }^{39}$ J. Draeger, ${ }^{39}$ H. Enderle, ${ }^{39}$ U. Gebbert, ${ }^{39}$ M. Görner, ${ }^{39}$ T. Hermanns, ${ }^{39}$ K. Kaschube, ${ }^{39}$ G. Kaussen, ${ }^{39}$ H. Kirschenmann, ${ }^{39}$ R. Klanner, ${ }^{39}$ J. Lange, ${ }^{39}$ B. Mura, ${ }^{39}$ F. Nowak, ${ }^{39}$ N. Pietsch, ${ }^{39}$ C. Sander, ${ }^{39}$ H. Schettler, ${ }^{39}$ P. Schleper, ${ }^{39}$ E. Schlieckau, ${ }^{39}$ M. Schröder ${ }^{39}$ T. Schum, ${ }^{39}$ H. Stadie, ${ }^{39}$ G. Steinbrück, ${ }^{39}$ J. Thomsen, ${ }^{39}$ C. Barth, ${ }^{40}$ J. Berger, ${ }^{40}$ T. Chwalek, ${ }^{40}$ W. De Boer, ${ }^{40}$ A. Dierlamm, ${ }^{40}$ G. Dirkes, ${ }^{40}$ M. Feindt, ${ }^{40}$ J. Gruschke, ${ }^{40}$ M. Guthoff, ${ }^{40, b}$ C. Hackstein, ${ }^{40}$ F. Hartmann, ${ }^{40}$ M. Heinrich, ${ }^{40}$ H. Held, ${ }^{40}$

K. H. Hoffmann, ${ }^{40}$ S. Honc, ${ }^{40}$ I. Katkov, ${ }^{40, n}$ J. R. Komaragiri, ${ }^{40}$ T. Kuhr, ${ }^{40}$ D. Martschei, ${ }^{40}$ S. Mueller, ${ }^{40}$ Th. Müller, ${ }^{40}$ M. Niegel, ${ }^{40}$ O. Oberst,${ }^{40}$ A. Oehler,${ }^{40}$ J. Ott, ${ }^{40}$ T. Peiffer, ${ }^{40}$ G. Quast,${ }^{40}$ K. Rabbertz, ${ }^{40}$ F. Ratnikov,${ }^{40}$ N. Ratnikova, ${ }^{40}$ M. Renz, ${ }^{40}$ S. Röcker, ${ }^{40}$ C. Saout, ${ }^{40}$ A. Scheurer, ${ }^{40}$ P. Schieferdecker, ${ }^{40}$ F.-P. Schilling, ${ }^{40}$ M. Schmanau, ${ }^{40}$

G. Schott, ${ }^{40}$ H. J. Simonis, ${ }^{40}$ F. M. Stober, ${ }^{40}$ D. Troendle,${ }^{40}$ J. Wagner-Kuhr,${ }^{40}$ T. Weiler, ${ }^{40}$ M. Zeise, ${ }^{40}$

E. B. Ziebarth, ${ }^{40}$ G. Daskalakis, ${ }^{41}$ T. Geralis ${ }^{41}$ S. Kesisoglou, ${ }^{41}$ A. Kyriakis, ${ }^{41}$ D. Loukas, ${ }^{41}$ I. Manolakos, ${ }^{41}$ A. Markou, ${ }^{41}$ C. Markou, ${ }^{41}$ C. Mavrommatis, ${ }^{41}$ E. Ntomari ${ }^{41}$ E. Petrakou, ${ }^{41}$ L. Gouskos, ${ }^{42}$ T. J. Mertzimekis ${ }^{42}$ A. Panagiotou, ${ }^{42}$ N. Saoulidou, ${ }^{42}$ E. Stiliaris, ${ }^{42}$ I. Evangelou, ${ }^{43}$ C. Foudas, ${ }^{43, b}$ P. Kokkas, ${ }^{43}$ N. Manthos, ${ }^{43}$ I. Papadopoulos, ${ }^{43}$ V. Patras, ${ }^{43}$ F. A. Triantis,${ }^{43}$ A. Aranyi,${ }^{44}$ G. Bencze, ${ }^{44}$ L. Boldizsar, ${ }^{44}$ C. Hajdu, ${ }^{44, b}$ P. Hidas, ${ }^{44}$ D. Horvath, ${ }^{44, p}$ A. Kapusi, ${ }^{44}$ K. Krajczar, ${ }^{44, q}$ F. Sikler, ${ }^{44, b}$ G. I. Veres, ${ }^{44, q}$ G. Vesztergombi, ${ }^{44, q}$ N. Beni, ${ }^{45}$ J. Molnar ${ }^{45}$ J. Palinkas, ${ }^{45}$ Z. Szillasi ${ }^{45}$ V. Veszpremi, ${ }^{45}$ J. Karancsi ${ }^{46}$ P. Raics, ${ }^{46}$ Z. L. Trocsanyi, ${ }^{46}$ B. Ujvari, ${ }^{46}$ S. B. Beri, ${ }^{47}$ V. Bhatnagar, ${ }^{47}$ N. Dhingra, ${ }^{47}$ R. Gupta, ${ }^{47}$ M. Jindal, ${ }^{47}$ M. Kaur, ${ }^{47}$ J. M. Kohli, ${ }^{47}$ M. Z. Mehta, ${ }^{47}$ N. Nishu, ${ }^{47}$ 
L. K. Saini,${ }^{47}$ A. Sharma, ${ }^{47}$ A. P. Singh, ${ }^{47}$ J. Singh,${ }^{47}$ S. P. Singh, ${ }^{47}$ S. Ahuja ${ }^{48}$ B. C. Choudhary, ${ }^{48}$ A. Kumar, ${ }^{48}$ A. Kumar ${ }^{48}$ S. Malhotra, ${ }^{48}$ M. Naimuddin, ${ }^{48}$ K. Ranjan, ${ }^{48}$ R. K. Shivpuri, ${ }^{48}$ S. Banerjee, ${ }^{49}$ S. Bhattacharya, ${ }^{49}$ S. Dutta, ${ }^{49}$ B. Gomber, ${ }^{49}$ S. Jain, ${ }^{49}$ S. Jain, ${ }^{49}$ R. Khurana,${ }^{49}$ S. Sarkar, ${ }^{49}$ R. K. Choudhury, ${ }^{50}$ D. Dutta, ${ }^{50}$ S. Kailas, ${ }^{50}$ V. Kumar, ${ }^{50}$ A. K. Mohanty, ${ }^{50, \mathrm{~b}}$ L. M. Pant,${ }^{50}$ P. Shukla, ${ }^{50}$ T. Aziz, ${ }^{51}$ M. Guchait, ${ }^{51, \mathrm{r}}$ A. Gurtu, ${ }^{51, \mathrm{~s}}$ M. Maity, ${ }^{51, \mathrm{~s}}$

D. Majumder, ${ }^{51}$ G. Majumder, ${ }^{51}$ K. Mazumdar, ${ }^{51}$ G. B. Mohanty, ${ }^{51}$ B. Parida,${ }^{51}$ A. Saha, ${ }^{51}$ K. Sudhakar,${ }^{51}$ N. Wickramage, ${ }^{51}$ S. Banerjee, ${ }^{52}$ S. Dugad, ${ }^{52}$ N. K. Mondal, ${ }^{52}$ H. Arfaei, ${ }^{53}$ H. Bakhshiansohi, ${ }^{53, \mathrm{t}}$ S. M. Etesami, ${ }^{53, \mathrm{u}}$ A. Fahim, ${ }^{53, \mathrm{t}}$ M. Hashemi, ${ }^{53}$ H. Hesari, ${ }^{53}$ A. Jafari, ${ }^{53, t}$ M. Khakzad, ${ }^{53}$ A. Mohammadi, ${ }^{53, v}$

M. Mohammadi Najafabadi, ${ }^{53}$ S. Paktinat Mehdiabadi, ${ }^{53}$ B. Safarzadeh,${ }^{53, w}$ M. Zeinali, ${ }^{53, u}$ M. Abbrescia,${ }^{54 a, 54 b}$ L. Barbone,${ }^{54 a, 54 b}$ C. Calabria, ${ }^{54 a, 54 b}$ A. Colaleo, ${ }^{54 a}$ D. Creanza, ${ }^{54 a, 54 c}$ N. De Filippis, ${ }^{54 a, 54 c, b}$ M. De Palma, ${ }^{54 a, 54 b}$ L. Fiore, ${ }^{54 \mathrm{a}}$ G. Iaselli, ${ }^{54 a, 54 \mathrm{c}}$ L. Lusito, ${ }^{54 a, 54 \mathrm{~b}}$ G. Maggi, ${ }^{54,54 \mathrm{c}}$ M. Maggi, ${ }^{54 \mathrm{a}}$ N. Manna, ${ }^{54 a, 54 \mathrm{~b}}$ B. Marangelli, ${ }^{54 a, 54 \mathrm{~b}}$ S. My, ${ }^{54 a, 54 c}$ S. Nuzzo, ${ }^{54 a, 54 b}$ N. Pacifico, ${ }^{54 a, 54 b}$ A. Pompili, ${ }^{54 a, 54 b}$ G. Pugliese,${ }^{54 a, 54 c}$ F. Romano, ${ }^{54 a, 54 c}$ G. Selvaggi, ${ }^{54 a, 54 b}$ L. Silvestris, ${ }^{54 a}$ S. Tupputi, ${ }^{54 a, 54 b}$ G. Zito,${ }^{54 a}$ G. Abbiendi, ${ }^{55 a}$ A. C. Benvenuti, ${ }^{55 a}$ D. Bonacorsi, ${ }^{55 a}$ S. Braibant-Giacomelli, ${ }^{55 a, 55 b}$ L. Brigliadori, ${ }^{55 a}$ P. Capiluppi,${ }^{55 a, 55 b}$ A. Castro, ${ }^{55 a, 55 b}$ F. R. Cavallo, ${ }^{55 a}$ M. Cuffiani ${ }^{55 a, 55 b}$ G. M. Dallavalle, ${ }^{55 a}$ F. Fabbri, ${ }^{55 a}$ A. Fanfani, ${ }^{55 a, 55 b}$ D. Fasanella, ${ }^{55 a, b}$ P. Giacomelli, ${ }^{55 a}$ C. Grandi, ${ }^{55 a}$ S. Marcellini, ${ }^{55 a}$ G. Masetti, ${ }^{55 a}$ M. Meneghelli, ${ }^{55 a, 55 b}$ A. Montanari, ${ }^{55 a}$ F. L. Navarria, ${ }^{55 a, 55 b}$ F. Odorici, ${ }^{55 a}$ A. Perrotta, ${ }^{55 a}$ F. Primavera, ${ }^{55 a}$ A. M. Rossi, ${ }^{55 a, 55 b}$ T. Rovelli, ${ }^{55 a, 55 b}$ G. Siroli, ${ }^{55 a, 55 b}$ R. Travaglini, ${ }^{55 a, 55 b}$ S. Albergo,${ }^{56 a, 56 b}$ G. Cappello, ${ }^{56 a, 56 b}$ M. Chiorboli, ${ }^{56 a, 56 b}$ S. Costa, ${ }^{56 a, 56 b}$ R. Potenza, ${ }^{56 a, 56 b}$ A. Tricomi, ${ }^{56 a, 56 b}$ C. Tuve, ${ }^{56 a, 56 b}$ G. Barbagli, ${ }^{57 a}$ V. Ciulli, ${ }^{57 a, 57 b}$ C. Civinini, ${ }^{57 a}$ R. D’Alessandro, ${ }^{57 a, 57 b}$ E. Focardi, ${ }^{57,57 b}$ S. Frosali, ${ }^{57,57 b}$ E. Gallo,${ }^{57 a}$ S. Gonzi,${ }^{57 a, 57 b}$ M. Meschini,${ }^{57 a}$ S. Paoletti, ${ }^{57 a}$ G. Sguazzoni, ${ }^{57 a}$ A. Tropiano, ${ }^{57 \mathrm{a}, \mathrm{b}}$ L. Benussi, ${ }^{58} \mathrm{~S}$. Bianco,${ }^{58} \mathrm{~S}$. Colafranceschi, ${ }^{58, \mathrm{x}}$ F. Fabbri,${ }^{58}$ D. Piccolo, ${ }^{58}$ P. Fabbricatore,${ }^{59}$ R. Musenich, ${ }^{59}$ A. Benaglia, ${ }^{60 a, 60 b, b}$ F. De Guio,${ }^{60 a, 60 b}$ L. Di Matteo,${ }^{60 a, 60 b}$ S. Gennai, ${ }^{60 a, b}$ A. Ghezzi, ${ }^{60 a, 60 b}$ S. Malvezzi, ${ }^{60 \mathrm{a}}$ A. Martelli, ${ }^{60 \mathrm{a}, 60 \mathrm{~b}}$ A. Massironi, ${ }^{60 \mathrm{a}, 60 \mathrm{~b}, \mathrm{~b}}$ D. Menasce, ${ }^{60 \mathrm{a}}$ L. Moroni, ${ }^{60 \mathrm{a}}$ M. Paganoni, ${ }^{60 \mathrm{a}, 60 \mathrm{~b}}$ D. Pedrini, ${ }^{60 \mathrm{a}}$ S. Ragazzi, ${ }^{60 a, 60 \mathrm{~b}}$ N. Redaelli, ${ }^{60 \mathrm{a}}$ S. Sala, ${ }^{60 \mathrm{a}}$ T. Tabarelli de Fatis,${ }^{60 \mathrm{a}, 60 \mathrm{~b}}$ S. Buontempo, ${ }^{61 \mathrm{a}}$

C. A. Carrillo Montoya, ${ }^{61 a, b}$ N. Cavallo, ${ }^{61 a, y}$ A. De Cosa,${ }^{61 a, 61 b}$ O. Dogangun, ${ }^{61 a, 61 b}$ F. Fabozzi, ${ }^{61 a, y}$ A. O. M. Iorio, ${ }^{61 a, b}$ L. Lista, ${ }^{61 a}$ M. Merola, ${ }^{61 a, 61 b}$ P. Paolucci, ${ }^{61 a}$ P. Azzi, ${ }^{62 a}$ N. Bacchetta, ${ }^{62 a, b}$ P. Bellan, ${ }^{62 a, 62 b}$ D. Bisello, ${ }^{62 a, 62 b}$ A. Branca, ${ }^{62 a}$ R. Carlin, ${ }^{62 a, 62 b}$ P. Checchia, ${ }^{62 a}$ T. Dorigo, ${ }^{62 a}$ U. Dosselli, ${ }^{62 a}$ F. Fanzago, ${ }^{62 a}$ F. Gasparini, ${ }^{62 a, 62 b}$ U. Gasparini, ${ }^{62 a, 62 b}$ A. Gozzelino, ${ }^{62 a}$ S. Lacaprara, ${ }^{62 a, z}$ I. Lazzizzera, ${ }^{62 a, 62 c}$ M. Margoni, ${ }^{62 a, 62 b}$ M. Mazzucato, ${ }^{62 a}$ A. T. Meneguzzo, ${ }^{62 a, 62 b}$ M. Nespolo, ${ }^{62 a, b}$ L. Perrozzi, ${ }^{62 a}$ N. Pozzobon, ${ }^{62 a, 62 b}$ P. Ronchese, ${ }^{62 a, 62 b}$ F. Simonetto, ${ }^{62 a, 62 b}$ E. Torassa, ${ }^{62 a}$ M. Tosi, ${ }^{62 a, 62 b, b}$ S. Vanini, ${ }^{62 a, 62 b}$ P. Zotto, ${ }^{62 a, 62 b}$ G. Zumerle, ${ }^{62 a, 62 b}$ P. Baesso, ${ }^{63 a, 63 b}$ U. Berzano, ${ }^{63 a}$ S. P. Ratti, ${ }^{63 a, 63 b}$ C. Riccardi, ${ }^{63 a, 63 b}$ P. Torre, ${ }^{63 a, 63 b}$ P. Vitulo, ${ }^{63 a, 63 b}$ C. Viviani, ${ }^{63 a, 63 b}$ M. Biasini, ${ }^{64 a, 64 b}$ G. M. Bilei, ${ }^{64 a}$ B. Caponeri, ${ }^{64 a, 64 b}$ L. Fanò, ${ }^{64 a, 64 b}$ P. Lariccia, ${ }^{64 a, 64 b}$ A. Lucaroni, ${ }^{64 a, 64 b, b}$ G. Mantovani, ${ }^{64 a, 64 b}$ M. Menichelli, ${ }^{64 a}$ A. Nappi, ${ }^{64 a, 64 b}$ F. Romeo, ${ }^{64 a, 64 b}$ A. Santocchia, ${ }^{64 a, 64 b}$ S. Taroni,,${ }^{64 a, 64 b, b}$ M. Valdata, ${ }^{64 a, 64 b}$ P. Azzurri, ${ }^{65 a, 65 c}$ G. Bagliesi, ${ }^{65 a}$ T. Boccali, ${ }^{65 a}$ G. Broccolo, ${ }^{65 a, 65 c}$ R. Castaldi, ${ }^{65 a}$ R. T. D’ Agnolo, ${ }^{65 a, 65 c}$ R. Dell'Orso, ${ }^{65 a}$ F. Fiori, ${ }^{65 a, 65 b}$ L. Foà, ${ }^{65 a, 65 c}$ A. Giassi, ${ }^{65 a}$ A. Kraan, ${ }^{65 a}$ F. Ligabue,${ }^{65 a, 65 c}$ T. Lomtadze,${ }^{65 a}$ L. Martini,${ }^{65 a, a a}$ A. Messineo, ${ }^{65 a, 65 b}$ F. Palla, ${ }^{65 a}$ F. Palmonari, ${ }^{65 a}$ A. Rizzi, ${ }^{65 a}$ G. Segneri, ${ }^{65 a}$ A. T. Serban, ${ }^{65 a}$ P. Spagnolo, ${ }^{65 a}$ R. Tenchini, ${ }^{65 a}$ G. Tonelli, ${ }^{65 a, 65 b, b}$ A. Venturi, ${ }^{65 a, b}$ P. G. Verdini,${ }^{65 a}$ L. Barone,${ }^{66 a, 66 b}$ F. Cavallari, ${ }^{66 a}$ D. Del Re,${ }^{66 a, 66 b, b}$ M. Diemoz, ${ }^{66 a}$ D. Franci, ${ }^{66 a, 66 b}$ M. Grassi, ${ }^{66 a, b}$ E. Longo, ${ }^{66 a, 66 b}$ P. Meridiani, ${ }^{66 a}$ S. Nourbakhsh ${ }^{66 a}$ G. Organtini, ${ }^{66 a, 66 b}$ F. Pandolfi, ${ }^{66 a, 66 b}$ R. Paramatti, ${ }^{66 a}$ S. Rahatlou, ${ }^{66 a, 66 b}$ M. Sigamani, ${ }^{66 a}$ N. Amapane, ${ }^{67 a, 67 b}$

R. Arcidiacono, ${ }^{67 a, 67 c}$ S. Argiro,${ }^{67,67 b}$ M. Arneodo, ${ }^{67 a, 67 c}$ C. Biino, ${ }^{67 a}$ C. Botta,${ }^{67 a, 67 b}$ N. Cartiglia, ${ }^{67 a}$ R. Castello, ${ }^{67 a, 67 b}$ M. Costa, ${ }^{67 a, 67 b}$ N. Demaria ${ }^{67 a}$ A. Graziano, ${ }^{67 a, 67 b}$ C. Mariotti, ${ }^{67 a, b}$ S. Maselli, ${ }^{67 a}$ E. Migliore, ${ }^{67 a, 67 b}$ V. Monaco, ${ }^{67 a, 67 b}$ M. Musich, ${ }^{67 a}$ M. M. Obertino, ${ }^{67 a, 67 c}$ N. Pastrone, ${ }^{67 a}$ M. Pelliccioni,${ }^{67 a}$ A. Potenza, ${ }^{67,67 b}$ A. Romero, ${ }^{67 a, 67 b}$ M. Ruspa,${ }^{67 a, 67 c}$ R. Sacchi, ${ }^{67 a, 67 b}$ V. Sola,${ }^{67 a, 67 b}$ A. Solano, ${ }^{67 a, 67 b}$ A. Staiano, ${ }^{67 a}$ A. Vilela Pereira, ${ }^{67 a}$ S. Belforte, ${ }^{68 a}$ F. Cossutti, ${ }^{68 a}$ G. Della Ricca, ${ }^{68 a, 68 b}$ B. Gobbo, ${ }^{68 a}$ M. Marone, ${ }^{68 a, 68 b}$ D. Montanino, ${ }^{68 \mathrm{a}, 68 \mathrm{~b}, \mathrm{~b}}$ A. Penzo, ${ }^{68 \mathrm{a}}$ S. G. Heo, ${ }^{69}$ S. K. Nam, ${ }^{69}$ S. Chang, ${ }^{70}$ J. Chung, ${ }^{70}$ D. H. Kim, ${ }^{70}$ G. N. Kim,${ }^{70}$ J. E. Kim ${ }^{70}$ D. J. Kong, ${ }^{70}$ H. Park, ${ }^{70}$ S. R. Ro, ${ }^{70}$ D. C. Son, ${ }^{70}$ T. Son, ${ }^{70}$ J. Y. Kim, ${ }^{71}$ Zero J. Kim, ${ }^{71}$ S. Song, ${ }^{71}$ H. Y. Jo, ${ }^{72}$ S. Choi, ${ }^{73}$ D. Gyun, ${ }^{73}$ B. Hong, ${ }^{73}$ M. Jo, ${ }^{73}$ H. Kim, ${ }^{73}$ T. J. Kim, ${ }^{73}$ K. S. Lee, ${ }^{73}$ D. H. Moon, ${ }^{73}$ S. K. Park, ${ }^{73}$ E. Seo, ${ }^{73}$ K. S. Sim,${ }^{73}$ M. Choi,${ }^{74}$ S. Kang,${ }^{74}$ H. Kim, ${ }^{74}$ J. H. Kim, ${ }^{74}$ C. Park, ${ }^{74}$ I. C. Park,${ }^{74}$ S. Park,${ }^{74}$ G. Ryu, ${ }^{74}$ Y. Cho, ${ }^{75}$ Y. Choi, ${ }^{75}$ Y. K. Choi, ${ }^{75}$ J. Goh, ${ }^{75}$ M. S. Kim, ${ }^{75}$ B. Lee, ${ }^{75}$ J. Lee, ${ }^{75}$ S. Lee, ${ }^{75}$ H. Seo, ${ }^{75}$ I. Yu, ${ }^{75}$ M. J. Bilinskas, ${ }^{76}$ I. Grigelionis, ${ }^{76}$ M. Janulis, ${ }^{76}$ D. Martisiute, ${ }^{76}$ P. Petrov,${ }^{76}$ M. Polujanskas, ${ }^{76}$ T. Sabonis, ${ }^{76}$ H. Castilla-Valdez, ${ }^{77}$ E. De La Cruz-Burelo,${ }^{77}$ I. Heredia-de La Cruz,${ }^{77}$ R. Lopez-Fernandez, ${ }^{77}$ R. Magaña Villalba, ${ }^{77}$ J. Martínez-Ortega, ${ }^{77}$ A. Sánchez-Hernández, ${ }^{77}$ L. M. Villasenor-Cendejas ${ }^{77}$ S. Carrillo Moreno, ${ }^{78}$ 
F. Vazquez Valencia,${ }^{78}$ H. A. Salazar Ibarguen, ${ }^{79}$ E. Casimiro Linares ${ }^{80}$ A. Morelos Pineda,${ }^{80}$ M. A. Reyes-Santos ${ }^{80}$ D. Krofcheck, ${ }^{81}$ A. J. Bell, ${ }^{82}$ P. H. Butler, ${ }^{82}$ R. Doesburg, ${ }^{82}$ S. Reucroft, ${ }^{82}$ H. Silverwood, ${ }^{82}$ M. Ahmad, ${ }^{83}$ M. I. Asghar, ${ }^{83}$ H. R. Hoorani, ${ }^{83}$ S. Khalid, ${ }^{83}$ W. A. Khan, ${ }^{83}$ T. Khurshid, ${ }^{83}$ S. Qazi, ${ }^{83}$ M. A. Shah, ${ }^{83}$ M. Shoaib ${ }^{83}$ G. Brona ${ }^{84}$ M. Cwiok, ${ }^{84}$ W. Dominik, ${ }^{84}$ K. Doroba, ${ }^{84}$ A. Kalinowski, ${ }^{84}$ M. Konecki, ${ }^{84}$ J. Krolikowski, ${ }^{84}$ H. Bialkowska, ${ }^{85}$ B. Boimska, ${ }^{85}$ T. Frueboes, ${ }^{85}$ R. Gokieli, ${ }^{85}$ M. Górski, ${ }^{85}$ M. Kazana ${ }^{85}$ K. Nawrocki, ${ }^{85}$

K. Romanowska-Rybinska, ${ }^{85}$ M. Szleper ${ }^{85}$ G. Wrochna, ${ }^{85}$ P. Zalewski, ${ }^{85}$ N. Almeida, ${ }^{86}$ P. Bargassa, ${ }^{86}$ A. David, ${ }^{86}$ P. Faccioli, ${ }^{86}$ P. G. Ferreira Parracho, ${ }^{86}$ M. Gallinaro, ${ }^{86}$ P. Musella, ${ }^{86}$ A. Nayak, ${ }^{86}$ J. Pela, ${ }^{86, b}$ P. Q. Ribeiro, ${ }^{86}$ J. Seixas, ${ }^{86}$ J. Varela, ${ }^{86}$ S. Afanasiev, ${ }^{87}$ I. Belotelov, ${ }^{87}$ P. Bunin,${ }^{87}$ M. Gavrilenko, ${ }^{87}$ I. Golutvin, ${ }^{87}$ I. Gorbunov, ${ }^{87}$ A. Kamenev, ${ }^{87}$ V. Karjavin, ${ }^{87}$ G. Kozlov ${ }^{87}$ A. Lanev, ${ }^{87}$ P. Moisenz, ${ }^{87}$ V. Palichik, ${ }^{87}$ V. Perelygin,${ }^{87}$ S. Shmatov, ${ }^{87}$ V. Smirnov, ${ }^{87}$ A. Volodko, ${ }^{87}$ A. Zarubin,${ }^{87}$ S. Evstyukhin, ${ }^{88}$ V. Golovtsov ${ }^{88}$ Y. Ivanov, ${ }^{88}$ V. Kim, ${ }^{88}$ P. Levchenko, ${ }^{88}$ V. Murzin, ${ }^{88}$ V. Oreshkin, ${ }^{88}$ I. Smirnov, ${ }^{88}$ V. Sulimov, ${ }^{88}$ L. Uvarov, ${ }^{88}$ S. Vavilov, ${ }^{88}$ A. Vorobyev ${ }^{88}$ An. Vorobyev, ${ }^{88}$ Yu. Andreev, ${ }^{89}$ A. Dermenev, ${ }^{89}$ S. Gninenko, ${ }^{89}$ N. Golubev,${ }^{89}$ M. Kirsanov, ${ }^{89}$ N. Krasnikov, ${ }^{89}$ V. Matveev, ${ }^{89}$ A. Pashenkov, ${ }^{89}$ A. Toropin, ${ }^{89}$ S. Troitsky, ${ }^{89}$ V. Epshteyn, ${ }^{90}$ M. Erofeeva, ${ }^{90}$ V. Gavrilov, ${ }^{90}$ M. Kossov, ${ }^{90, b}$ A. Krokhotin, ${ }^{90}$ N. Lychkovskaya, ${ }^{90}$ V. Popov, ${ }^{90}$ G. Safronov, ${ }^{90}$ S. Semenov, ${ }^{90}$ V. Stolin,${ }^{90}$ E. Vlasov, ${ }^{90}$ A. Zhokin, ${ }^{90}$ A. Belyaev, ${ }^{91}$ E. Boos,${ }^{91}$ M. Dubinin, ${ }^{91, e}$ L. Dudko, ${ }^{91}$ A. Ershov, ${ }^{91}$ A. Gribushin, ${ }^{91}$ O. Kodolova, ${ }^{91}$ I. Lokhtin,${ }^{91}$ A. Markina, ${ }^{91}$ S. Obraztsov,${ }^{91}$ M. Perfilov, ${ }^{91}$ S. Petrushanko, ${ }^{91}$ L. Sarycheva, ${ }^{91}$ V. Savrin, ${ }^{91}$ A. Snigirev, ${ }^{91}$ V. Andreev, ${ }^{92}$ M. Azarkin, ${ }^{92}$ I. Dremin, ${ }^{92}$ M. Kirakosyan, ${ }^{92}$ A. Leonidov,${ }^{92}$ G. Mesyats, ${ }^{92}$ S. V. Rusakov, ${ }^{92}$ A. Vinogradov, ${ }^{92}$ I. Azhgirey, ${ }^{93}$ I. Bayshev, ${ }^{93}$ S. Bitioukov, ${ }^{93}$ V. Grishin, ${ }^{93, b}$ V. Kachanov, ${ }^{93}$ D. Konstantinov, ${ }^{93}$ A. Korablev, ${ }^{93}$ V. Krychkine, ${ }^{93}$ V. Petrov,${ }^{93}$ R. Ryutin, ${ }^{93}$ A. Sobol,${ }^{93}$ L. Tourtchanovitch, ${ }^{93}$ S. Troshin,${ }^{93}$ N. Tyurin, ${ }^{93}$ A. Uzunian, ${ }^{93}$ A. Volkov, ${ }^{93}$ P. Adzic,${ }^{94, b b}$ M. Djordjevic,,${ }^{94}$ M. Ekmedzic, ${ }^{94}$ D. Krpic, ${ }^{94, b b}$ J. Milosevic,${ }^{94}$ M. Aguilar-Benitez,${ }^{95}$ J. Alcaraz Maestre, ${ }^{95}$ P. Arce, ${ }^{95}$ C. Battilana,${ }^{95}$ E. Calvo, ${ }^{95}$ M. Cerrada,${ }^{95}$

M. Chamizo Llatas, ${ }^{95}$ N. Colino, ${ }^{95}$ B. De La Cruz, ${ }^{95}$ A. Delgado Peris,${ }^{95}$ C. Diez Pardos, ${ }^{95}$ D. Domínguez Vázquez, ${ }^{95}$ C. Fernandez Bedoya,${ }^{95}$ J. P. Fernández Ramos, ${ }^{95}$ A. Ferrando, ${ }^{95}$ J. Flix,${ }^{95}$ M. C. Fouz,${ }^{95}$ P. Garcia-Abia, ${ }^{95}$ O. Gonzalez Lopez,${ }^{95}$ S. Goy Lopez, ${ }^{95}$ J. M. Hernandez, ${ }^{95}$ M. I. Josa, ${ }^{95}$ G. Merino, ${ }^{95}$ J. Puerta Pelayo,${ }^{95}$ I. Redondo, ${ }^{95}$ L. Romero, ${ }^{95}$ J. Santaolalla, ${ }^{95}$ M. S. Soares, ${ }^{95}$ C. Willmott, ${ }^{95}$ C. Albajar, ${ }^{96}$ G. Codispoti,,${ }^{96}$ J. F. de Trocóniz, ${ }^{96}$ J. Cuevas, ${ }^{97}$ J. Fernandez Menendez, ${ }^{97}$ S. Folgueras, ${ }^{97}$ I. Gonzalez Caballero, ${ }^{97}$

L. Lloret Iglesias, ${ }^{97}$ J. M. Vizan Garcia, ${ }^{97}$ J. A. Brochero Cifuentes, ${ }^{98}$ I. J. Cabrillo, ${ }^{98}$ A. Calderon, ${ }^{98}$ S. H. Chuang, ${ }^{98}$

J. Duarte Campderros, ${ }^{98}$ M. Felcini, ${ }^{98, c c}$ M. Fernandez, ${ }^{98}$ G. Gomez, ${ }^{98}$ J. Gonzalez Sanchez,${ }^{98}$ C. Jorda, ${ }^{98}$

P. Lobelle Pardo, ${ }^{98}$ A. Lopez Virto,${ }^{98}$ J. Marco,${ }^{98}$ R. Marco,${ }^{98}$ C. Martinez Rivero, ${ }^{98}$ F. Matorras,${ }^{98}$

F. J. Munoz Sanchez, ${ }^{98}$ J. Piedra Gomez,${ }^{98, d d}$ T. Rodrigo, ${ }^{98}$ A. Y. Rodríguez-Marrero, ${ }^{98}$ A. Ruiz-Jimeno, ${ }^{98}$ L. Scodellaro, ${ }^{98}$ M. Sobron Sanudo, ${ }^{98}$ I. Vila, ${ }^{98}$ R. Vilar Cortabitarte, ${ }^{98}$ D. Abbaneo, ${ }^{99}$ E. Auffray,${ }^{99}$ G. Auzinger,${ }^{99}$ P. Baillon, ${ }^{99}$ A. H. Ball, ${ }^{99}$ D. Barney, ${ }^{99}$ C. Bernet, ${ }^{99, f}$ W. Bialas, ${ }^{99}$ P. Bloch,${ }^{99}$ A. Bocci, ${ }^{99}$ H. Breuker, ${ }^{99}$ K. Bunkowski, ${ }^{99}$ T. Camporesi, ${ }^{99}$ G. Cerminara, ${ }^{99}$ T. Christiansen, ${ }^{99}$ J. A. Coarasa Perez, ${ }^{99}$ B. Curé, ${ }^{99}$ D. D'Enterria, ${ }^{99}$ A. De Roeck, ${ }^{99}$ S. Di Guida, ${ }^{99}$ M. Dobson, ${ }^{99}$ N. Dupont-Sagorin, ${ }^{99}$ A. Elliott-Peisert, ${ }^{99}$ B. Frisch, ${ }^{99}$ W. Funk,${ }^{99}$ A. Gaddi, ${ }^{99}$ G. Georgiou, ${ }^{99}$ H. Gerwig, ${ }^{99}$ M. Giffels, ${ }^{99}$ D. Gigi,${ }^{99}$ K. Gill, ${ }^{99}$ D. Giordano, ${ }^{99}$ M. Giunta,${ }^{99}$ F. Glege,${ }^{99}$ R. Gomez-Reino Garrido, ${ }^{99}$ P. Govoni,${ }^{99}$ S. Gowdy, ${ }^{99}$ R. Guida,${ }^{99}$ L. Guiducci, ${ }^{99}$ S. Gundacker, ${ }^{99}$ M. Hansen, ${ }^{99}$ C. Hartl, ${ }^{99}$ J. Harvey, ${ }^{99}$ J. Hegeman, ${ }^{99}$ B. Hegner, ${ }^{99}$ H. F. Hoffmann,${ }^{99}$ V. Innocente, ${ }^{99}$ P. Janot, ${ }^{99}$ K. Kaadze, ${ }^{99}$ E. Karavakis, ${ }^{99}$ P. Lecoq,${ }^{99}$ P. Lenzi,${ }^{99}$ C. Lourenço, ${ }^{99}$ T. Mäki, ${ }^{99}$ M. Malberti, ${ }^{99}$ L. Malgeri, ${ }^{99}$ M. Mannelli, ${ }^{99}$ L. Masetti, ${ }^{99}$ G. Mavromanolakis, ${ }^{99}$ F. Meijers, ${ }^{99}$ S. Mersi,${ }^{99}$ E. Meschi, ${ }^{99}$ R. Moser, ${ }^{99}$ M. U. Mozer, ${ }^{99}$ M. Mulders, ${ }^{99}$ E. Nesvold,${ }^{99}$ M. Nguyen, ${ }^{99}$ T. Orimoto, ${ }^{99}$ L. Orsini, ${ }^{99}$ E. Palencia Cortezon, ${ }^{99}$ E. Perez ${ }^{99}$ A. Petrilli, ${ }^{99}$ A. Pfeiffer,${ }^{99}$ M. Pierini,,${ }^{99}$ M. Pimiä, ${ }^{99}$ D. Piparo, ${ }^{99}$ G. Polese,${ }^{99}$ L. Quertenmont, ${ }^{99}$

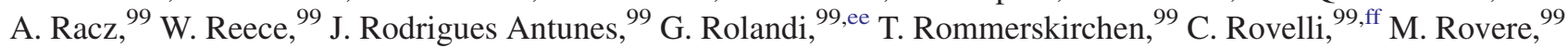
H. Sakulin, ${ }^{99}$ F. Santanastasio, ${ }^{99}$ C. Schäfer, ${ }^{99}$ C. Schwick, ${ }^{99}$ I. Segoni, ${ }^{99}$ A. Sharma, ${ }^{99}$ P. Siegrist, ${ }^{99}$ P. Silva, ${ }^{99}$ M. Simon, ${ }^{99}$ P. Sphicas, ${ }^{99, g g}$ D. Spiga,${ }^{99}$ M. Spiropulu, ${ }^{99, \mathrm{e}}$ M. Stoye,${ }^{99}$ A. Tsirou, ${ }^{99}$ P. Vichoudis, ${ }^{99}$ H. K. Wöhri, ${ }^{99}$ S. D. Worm, ${ }^{99, \text { hh }}$ W. D. Zeuner, ${ }^{99}$ W. Bertl, ${ }^{100}$ K. Deiters, ${ }^{100}$ W. Erdmann, ${ }^{100}$ K. Gabathuler ${ }^{100}$ R. Horisberger, ${ }^{100}$ Q. Ingram, ${ }^{100}$ H. C. Kaestli, ${ }^{100}$ S. König, ${ }^{100}$ D. Kotlinski, ${ }^{100}$ U. Langenegger, ${ }^{100}$ F. Meier,${ }^{100}$ D. Renker,${ }^{100}$ T. Rohe, ${ }^{100}$ J. Sibille, ${ }^{100, \text { ii }}$ L. Bäni, ${ }^{101}$ P. Bortignon, ${ }^{101}$ B. Casal, ${ }^{101}$ N. Chanon, ${ }^{101}$ Z. Chen, ${ }^{101}$ S. Cittolin, ${ }^{101}$ A. Deisher, ${ }^{101}$ G. Dissertori, ${ }^{101}$ M. Dittmar, ${ }^{101}$ J. Eugster, ${ }^{101}$ K. Freudenreich,${ }^{101}$ C. Grab,${ }^{101}$ P. Lecomte,${ }^{101}$ W. Lustermann, ${ }^{101}$ P. Martinez Ruiz del Arbol, ${ }^{101}$ P. Milenovic, ${ }^{101, j \mathrm{jj}}$ N. Mohr, ${ }^{101}$ F. Moortgat,${ }^{101}$ C. Nägeli, ${ }^{101, k k}$ P. Nef, ${ }^{101}$ F. Nessi-Tedaldi, ${ }^{101}$ L. Pape,${ }^{101}$ F. Pauss, ${ }^{101}$ M. Peruzzi,${ }^{101}$ F. J. Ronga,${ }^{101}$ M. Rossini, ${ }^{101}$ L. Sala, ${ }^{101}$ A. K. Sanchez, ${ }^{101}$ M.-C. Sawley, ${ }^{101}$ A. Starodumov, ${ }^{101,11}$ B. Stieger,${ }^{101}$ M. Takahashi, ${ }^{101}$ L. Tauscher, ${ }^{101, a}$ A. Thea, ${ }^{101}$ 
K. Theofilatos, ${ }^{101}$ D. Treille, ${ }^{101}$ C. Urscheler, ${ }^{101}$ R. Wallny, ${ }^{101}$ H. A. Weber,${ }^{101}$ L. Wehrli, ${ }^{101}$ J. Weng, ${ }^{101}$ E. Aguilo, ${ }^{102}$ C. Amsler, ${ }^{102}$ V. Chiochia, ${ }^{102}$ S. De Visscher, ${ }^{102}$ C. Favaro,${ }^{102}$ M. Ivova Rikova, ${ }^{102}$ B. Millan Mejias,${ }^{102}$ P. Otiougova, ${ }^{102}$ P. Robmann, ${ }^{102}$ A. Schmidt, ${ }^{102}$ H. Snoek, ${ }^{102}$ M. Verzetti, ${ }^{102}$ Y. H. Chang, ${ }^{103}$ K. H. Chen, ${ }^{103}$

C. M. Kuo, ${ }^{103}$ S. W. Li, ${ }^{103}$ W. Lin, ${ }^{103}$ Z. K. Liu, ${ }^{103}$ Y. J. Lu, ${ }^{103}$ D. Mekterovic, ${ }^{103}$ R. Volpe, ${ }^{103}$ S. S. Yu, ${ }^{103}$ P. Bartalini, ${ }^{104}$ P. Chang, ${ }^{104}$ Y. H. Chang, ${ }^{104}$ Y. W. Chang, ${ }^{104}$ Y. Chao, ${ }^{104}$ K. F. Chen, ${ }^{104}$ C. Dietz, ${ }^{104}$ U. Grundler, ${ }^{104}$ W.-S. Hou, ${ }^{104}$ Y. Hsiung, ${ }^{104}$ K. Y. Kao, ${ }^{104}$ Y. J. Lei, ${ }^{104}$ R.-S. Lu, ${ }^{104}$ J. G. Shiu, ${ }^{104}$ Y. M. Tzeng, ${ }^{104}$ X. Wan, ${ }^{104}$ M. Wang, ${ }^{104}$ A. Adiguzel, ${ }^{105}$ M. N. Bakirci, ${ }^{105, \mathrm{~mm}}$ S. Cerci, ${ }^{105, \mathrm{nn}}$ C. Dozen, ${ }^{105}$ I. Dumanoglu, ${ }^{105}$ E. Eskut, ${ }^{105}$ S. Girgis, ${ }^{105}$ G. Gokbulut, ${ }^{105}$ I. Hos, ${ }^{105}$ E. E. Kangal, ${ }^{105}$ G. Karapinar, ${ }^{105}$ A. Kayis Topaksu, ${ }^{105}$ G. Onengut, ${ }^{105}$

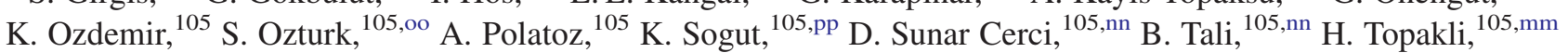
D. Uzun, ${ }^{105}$ L. N. Vergili, ${ }^{105}$ M. Vergili, ${ }^{105}$ I. V. Akin, ${ }^{106}$ T. Aliev,${ }^{106}$ B. Bilin, ${ }^{106}$ S. Bilmis,${ }^{106}$ M. Deniz, ${ }^{106}$ H. Gamsizkan, ${ }^{106}$ A. M. Guler, ${ }^{106}$ K. Ocalan, ${ }^{106}$ A. Ozpineci, ${ }^{106}$ M. Serin, ${ }^{106}$ R. Sever, ${ }^{106}$ U. E. Surat, ${ }^{106}$ M. Yalvac, ${ }^{106}$ E. Yildirim, ${ }^{106}$ M. Zeyrek, ${ }^{106}$ M. Deliomeroglu ${ }^{107}$ E. Gülmez, ${ }^{107}$ B. Isildak, ${ }^{107}$ M. Kaya, ${ }^{107, q q}$ O. Kaya, ${ }^{107, q q}$ M. Özbek, ${ }^{107}$ S. Ozkorucuklu, ${ }^{107, \text { rr }}$ N. Sonmez, ${ }^{107, \text { ss }}$ L. Levchuk, ${ }^{108}$ F. Bostock, ${ }^{109}$ J. J. Brooke, ${ }^{109}$ E. Clement, ${ }^{109}$ D. Cussans, ${ }^{109}$ R. Frazier, ${ }^{109}$ J. Goldstein, ${ }^{109}$ M. Grimes, ${ }^{109}$ G. P. Heath ${ }^{109}$ H. F. Heath,${ }^{109}$ L. Kreczko, ${ }^{109}$ S. Metson, ${ }^{109}$ D. M. Newbold,${ }^{109, \text { hh }}$ K. Nirunpong, ${ }^{109}$ A. Poll, ${ }^{109}$ S. Senkin, ${ }^{109}$ V. J. Smith,${ }^{109}$ T. Williams, ${ }^{109}$ L. Basso, ${ }^{110, t \mathrm{tt}}$ K. W. Bell, ${ }^{110}$ A. Belyaev, ${ }^{110, t \mathrm{t}}$ C. Brew, ${ }^{110}$ R. M. Brown, ${ }^{110}$ B. Camanzi, ${ }^{110}$ D. J. A. Cockerill, ${ }^{110}$ J. A. Coughlan, ${ }^{110}$ K. Harder,${ }^{10}$ S. Harper, ${ }^{110}$ J. Jackson, ${ }^{110}$ B. W. Kennedy, ${ }^{110}$ E. Olaiya, ${ }^{110}$ D. Petyt, ${ }^{110}$ B. C. Radburn-Smith, ${ }^{110}$ C. H. Shepherd-Themistocleous, ${ }^{110}$ I. R. Tomalin, ${ }^{110}$ W. J. Womersley, ${ }^{110}$ R. Bainbridge, ${ }^{111}$ G. Ball, ${ }^{111}$ R. Beuselinck, ${ }^{111}$ O. Buchmuller, ${ }^{111}$ D. Colling, ${ }^{111}$ N. Cripps, ${ }^{111}$ M. Cutajar, ${ }^{111}$ P. Dauncey, ${ }^{111}$ G. Davies, ${ }^{111}$ M. Della Negra, ${ }^{111}$ W. Ferguson,${ }^{111}$ J. Fulcher, ${ }^{111}$ D. Futyan, ${ }^{111}$ A. Gilbert, ${ }^{111}$ A. Guneratne Bryer, ${ }^{111}$ G. Hall, ${ }^{111}$ Z. Hatherell, ${ }^{111}$ J. Hays, ${ }^{111}$ G. Iles, ${ }^{111}$ M. Jarvis, ${ }^{111}$ G. Karapostoli,,${ }^{111}$ L. Lyons, ${ }^{111}$ A.-M. Magnan, ${ }^{111}$ J. Marrouche,${ }^{111}$ B. Mathias, ${ }^{111}$ R. Nandi,,${ }^{11}$ J. Nash, ${ }^{111}$ A. Nikitenko, ${ }^{111,11}$

A. Papageorgiou, ${ }^{111}$ M. Pesaresi, ${ }^{111}$ K. Petridis, ${ }^{111}$ M. Pioppi, ${ }^{111, \text { uu }}$ D. M. Raymond, ${ }^{111}$ S. Rogerson,,${ }^{111}$ N. Rompotis, ${ }^{111}$ A. Rose, ${ }^{111}$ M. J. Ryan, ${ }^{111}$ C. Seez, ${ }^{111}$ P. Sharp, ${ }^{111}$ A. Sparrow, ${ }^{111}$ A. Tapper, ${ }^{111}$ S. Tourneur, ${ }^{111}$ M. Vazquez Acosta, ${ }^{111}$ T. Virdee, ${ }^{111}$ S. Wakefield, ${ }^{111}$ N. Wardle, ${ }^{111}$ D. Wardrope, ${ }^{111}$ T. Whyntie, ${ }^{111}$ M. Barrett, ${ }^{112}$ M. Chadwick, ${ }^{112}$ J. E. Cole, ${ }^{112}$ P. R. Hobson, ${ }^{112}$ A. Khan, ${ }^{112}$ P. Kyberd, ${ }^{112}$ D. Leslie, ${ }^{112}$ W. Martin, ${ }^{112}$ I. D. Reid, ${ }^{112}$ L. Teodorescu, ${ }^{112}$ M. Turner, ${ }^{112}$ K. Hatakeyama, ${ }^{113}$ H. Liu, ${ }^{113}$ T. Scarborough, ${ }^{113}$ C. Henderson, ${ }^{114}$ A. Avetisyan, ${ }^{115}$ T. Bose, ${ }^{115}$ E. Carrera Jarrin, ${ }^{115}$ C. Fantasia, ${ }^{115}$ A. Heister, ${ }^{115}$ J. St. John, ${ }^{115}$ P. Lawson, ${ }^{15}$ D. Lazic, ${ }^{115}$ J. Rohlf, ${ }^{115}$ D. Sperka, ${ }^{115}$ L. Sulak, ${ }^{115}$ S. Bhattacharya, ${ }^{116}$ D. Cutts, ${ }^{116}$ A. Ferapontov, ${ }^{116}$ U. Heintz, ${ }^{116}$ S. Jabeen, ${ }^{116}$

G. Kukartsev, ${ }^{116}$ G. Landsberg, ${ }^{116}$ M. Luk, ${ }^{116}$ M. Narain, ${ }^{116}$ D. Nguyen, ${ }^{116}$ M. Segala, ${ }^{116}$ T. Sinthuprasith, ${ }^{116}$ T. Speer, ${ }^{116}$ K. V. Tsang, ${ }^{116}$ R. Breedon, ${ }^{117}$ G. Breto, ${ }^{117}$ M. Calderon De La Barca Sanchez, ${ }^{17}$ S. Chauhan, ${ }^{117}$ M. Chertok,,${ }^{117}$ J. Conway, ${ }^{117}$ R. Conway, ${ }^{117}$ P. T. Cox,${ }^{117}$ J. Dolen, ${ }^{117}$ R. Erbacher,${ }^{117}$ R. Houtz,${ }^{117}$ W. Ko, ${ }^{117}$ A. Kopecky, ${ }_{117}^{117}$ R. Lander, ${ }^{117}$ O. Mall, ${ }^{117}$ T. Miceli, ${ }^{117}$ D. Pellett, ${ }^{117}$ J. Robles, ${ }^{117}$ B. Rutherford, ${ }^{117}$ M. Searle, ${ }^{117}$ J. Smith, ${ }^{117}$ M. Squires,${ }^{117}$ M. Tripathi, ${ }^{117}$ R. Vasquez Sierra, ${ }^{117}$ V. Andreev,${ }^{118}$ K. Arisaka, ${ }^{118}$ D. Cline,${ }^{118}$ R. Cousins, ${ }^{118}$ J. Duris, ${ }^{118}$ S. Erhan, ${ }^{118}$ P. Everaerts, ${ }^{118}$ C. Farrell, ${ }^{118}$ J. Hauser, ${ }^{118}$ M. Ignatenko, ${ }^{118}$ C. Jarvis,${ }^{118}$ C. Plager, ${ }^{118}$ G. Rakness, ${ }^{118}$ P. Schlein, ${ }^{118, a}$ J. Tucker, ${ }^{11}$ V. Valuev, ${ }^{18}$ M. Weber, ${ }^{18}$ J. Babb,${ }^{19}$ R. Clare, ${ }^{119}$ J. Ellison, ${ }^{119}$ J. W. Gary, ${ }^{119}$ F. Giordano, ${ }^{119}$ G. Hanson, ${ }^{119}$ G. Y. Jeng, ${ }^{119}$ S. C. Kao, ${ }^{119}$ H. Liu, ${ }^{119}$ O. R. Long, ${ }^{119}$ A. Luthra, ${ }^{119}$ H. Nguyen, ${ }^{119}$ S. Paramesvaran, ${ }^{119}$ J. Sturdy, ${ }^{119}$ S. Sumowidagdo, ${ }^{119}$ R. Wilken, ${ }^{19}$ S. Wimpenny, ${ }^{119}$ W. Andrews, ${ }^{120}$ J. G. Branson, ${ }^{120}$ G. B. Cerati, ${ }^{120}$ D. Evans, ${ }^{120}$ F. Golf, ${ }^{120}$ A. Holzner, ${ }^{120}$ R. Kelley,${ }^{120}$

M. Lebourgeois, ${ }^{120}$ J. Letts, ${ }^{120}$ I. Macneill, ${ }^{120}$ B. Mangano, ${ }^{120}$ S. Padhi, ${ }^{120}$ C. Palmer, ${ }^{120}$ G. Petrucciani, ${ }^{120}$ H. Pi,${ }^{120}$ M. Pieri, ${ }^{120}$ R. Ranieri, ${ }^{120}$ M. Sani, ${ }^{120}$ I. Sfiligoi, ${ }^{120}$ V. Sharma, ${ }^{120}$ S. Simon, ${ }^{120}$ E. Sudano, ${ }^{120}$ M. Tadel,,${ }^{120}$ Y. Tu, ${ }^{120}$ A. Vartak, ${ }^{120}$ S. Wasserbaech, ${ }^{120, v v}$ F. Würthwein, ${ }^{120}$ A. Yagil,${ }^{120}$ J. Yoo, ${ }^{120}$ D. Barge,${ }^{121}$ R. Bellan, ${ }^{121}$

C. Campagnari, ${ }^{121}$ M. D'Alfonso, ${ }^{121}$ T. Danielson, ${ }^{121}$ K. Flowers, ${ }^{121}$ P. Geffert,${ }^{121}$ C. George,${ }^{121}$ J. Incandela, ${ }^{121}$ C. Justus, ${ }^{121}$ P. Kalavase, ${ }^{121}$ S. A. Koay, ${ }^{121}$ D. Kovalskyi, ${ }^{121, b}$ V. Krutelyov, ${ }^{121}$ S. Lowette, ${ }^{121}$ N. Mccoll, ${ }^{121}$ S. D. Mullin, ${ }^{121}$ V. Pavlunin, ${ }^{121}$ F. Rebassoo, ${ }^{121}$ J. Ribnik, ${ }^{121}$ J. Richman, ${ }^{121}$ R. Rossin, ${ }^{121}$ D. Stuart, ${ }^{121}$ W. To, ${ }^{121}$ J. R. Vlimant, ${ }^{121}$ C. West, ${ }^{121}$ A. Apresyan, ${ }^{122}$ A. Bornheim, ${ }^{122}$ J. Bunn, ${ }^{122}$ Y. Chen, ${ }^{122}$ E. Di Marco, ${ }^{122}$ J. Duarte, ${ }^{122}$ M. Gataullin, ${ }^{122}$ Y. Ma, ${ }^{122}$ A. Mott, ${ }^{122}$ H. B. Newman, ${ }^{122}$ C. Rogan, ${ }^{122}$ V. Timciuc, ${ }^{122}$ P. Traczyk, ${ }^{122}$ J. Veverka, ${ }^{122}$ R. Wilkinson, ${ }^{122}$ Y. Yang, ${ }^{122}$ R. Y. Zhu, ${ }^{122}$ B. Akgun, ${ }^{123}$ R. Carroll, ${ }^{123}$ T. Ferguson, ${ }^{123}$ Y. Iiyama, ${ }^{123}$ D. W. Jang, ${ }^{123}$ S. Y. Jun, ${ }^{123}$ Y. F. Liu, ${ }^{123}$ M. Paulini, ${ }^{123}$ J. Russ, ${ }^{123}$ H. Vogel, ${ }^{123}$ I. Vorobiev, ${ }^{123}$ J. P. Cumalat, ${ }^{124}$ M. E. Dinardo, ${ }^{124}$ B. R. Drell, ${ }^{124}$ C. J. Edelmaier, ${ }^{124}$ W. T. Ford, ${ }^{124}$ A. Gaz, ${ }^{124}$ B. Heyburn, ${ }^{124}$ E. Luiggi Lopez, ${ }^{124}$ U. Nauenberg, ${ }^{124}$ J. G. Smith, ${ }^{124}$ K. Stenson, ${ }^{124}$ K. A. Ulmer, ${ }^{124}$ S. R. Wagner, ${ }^{124}$ S. L. Zang, ${ }^{124}$ L. Agostino, ${ }^{125}$ J. Alexander, ${ }^{125}$ 
A. Chatterjee, ${ }^{125}$ N. Eggert, ${ }^{125}$ L. K. Gibbons, ${ }^{125}$ B. Heltsley, ${ }^{125}$ W. Hopkins, ${ }^{125}$ A. Khukhunaishvili, ${ }^{125}$ B. Kreis, ${ }^{125}$ G. Nicolas Kaufman, ${ }^{125}$ J. R. Patterson, ${ }^{125}$ D. Puigh, ${ }^{125}$ A. Ryd, ${ }^{125}$ E. Salvati, ${ }^{125}$ X. Shi, ${ }^{125}$ W. Sun,${ }^{125}$ W. D. Teo, ${ }^{125}$ J. Thom, ${ }^{125}$ J. Thompson, ${ }^{125}$ J. Vaughan, ${ }^{125}$ Y. Weng, ${ }^{125}$ L. Winstrom, ${ }^{125}$ P. Wittich, ${ }^{125}$ A. Biselli, ${ }^{126}$ G. Cirino, ${ }^{126}$ D. Winn, ${ }^{126}$ S. Abdullin, ${ }^{127}$ M. Albrow, ${ }^{127}$ J. Anderson, ${ }^{127}$ G. Apollinari, ${ }^{127}$ M. Atac, ${ }^{127}$ J. A. Bakken, ${ }^{127}$

L. A. T. Bauerdick, ${ }^{127}$ A. Beretvas, ${ }^{127}$ J. Berryhill, ${ }^{127}$ P. C. Bhat, ${ }^{127}$ I. Bloch, ${ }^{127}$ K. Burkett, ${ }^{127}$ J. N. Butler, ${ }^{127}$ V. Chetluru, ${ }^{127}$ H. W. K. Cheung, ${ }^{127}$ F. Chlebana, ${ }^{127}$ S. Cihangir, ${ }^{127}$ W. Cooper, ${ }^{127}$ D. P. Eartly, ${ }^{127}$ V. D. Elvira, ${ }^{127}$ S. Esen, ${ }^{127}$ I. Fisk, ${ }^{127}$ J. Freeman, ${ }^{127}$ Y. Gao, ${ }^{127}$ E. Gottschalk, ${ }^{127}$ D. Green, ${ }^{127}$ O. Gutsche, ${ }^{127}$ J. Hanlon, ${ }^{127}$ R. M. Harris, ${ }^{127}$ J. Hirschauer, ${ }^{127}$ B. Hooberman, ${ }^{127}$ H. Jensen, ${ }^{127}$ S. Jindariani, ${ }^{127}$ M. Johnson, ${ }^{127}$ U. Joshi, ${ }^{127}$ B. Klima, ${ }^{127}$ K. Kousouris, ${ }^{127}$ S. Kunori, ${ }^{127}$ S. Kwan, ${ }^{127}$ C. Leonidopoulos, ${ }^{127}$ D. Lincoln, ${ }^{127}$ R. Lipton, ${ }^{127}$ J. Lykken, ${ }^{127}$ K. Maeshima, ${ }^{127}$ J. M. Marraffino, ${ }^{127}$ S. Maruyama, ${ }^{127}$ D. Mason, ${ }^{127}$ P. McBride, ${ }^{127}$ T. Miao, ${ }^{127}$ K. Mishra, ${ }^{127}$ S. Mrenna, ${ }^{127}$ Y. Musienko, ${ }^{127, w w}$ C. Newman-Holmes, ${ }^{127}$ V. O’Dell, ${ }^{127}$ J. Pivarski, ${ }^{127}$ R. Pordes, ${ }^{127}$ O. Prokofyev, ${ }^{127}$ T. Schwarz, ${ }^{127}$ E. Sexton-Kennedy, ${ }^{127}$ S. Sharma, ${ }^{127}$ W. J. Spalding, ${ }^{127}$ L. Spiegel, ${ }^{127}$ P. Tan,${ }^{127}$ L. Taylor, ${ }^{127}$ S. Tkaczyk, ${ }^{127}$ L. Uplegger, ${ }^{127}$ E. W. Vaandering, ${ }^{127}$ R. Vidal, ${ }^{127}$ J. Whitmore,,${ }^{127}$ W. Wu, ${ }^{127}$ F. Yang, ${ }^{127}$ F. Yumiceva, ${ }^{127}$ J. C. Yun, ${ }^{127}$ D. Acosta, ${ }^{128}$ P. Avery, ${ }^{128}$ D. Bourilkov, ${ }^{128}$ M. Chen, ${ }^{128}$ S. Das, ${ }^{128}$ M. De Gruttola, ${ }^{128}$ G. P. Di Giovanni, ${ }^{128}$ D. Dobur, ${ }^{128}$ A. Drozdetskiy, ${ }^{128}$ R. D. Field, ${ }^{128}$ M. Fisher, ${ }^{128}$ Y. Fu, ${ }^{128}$ I. K. Furic, ${ }^{128}$ J. Gartner, ${ }^{128}$ S. Goldberg, ${ }^{128}$ J. Hugon, ${ }^{128}$ B. Kim, ${ }^{128}$ J. Konigsberg, ${ }^{128}$ A. Korytov, ${ }^{128}$ A. Kropivnitskaya, ${ }^{128}$ T. Kypreos, ${ }^{128}$ J. F. Low, ${ }^{128}$ K. Matchev, ${ }^{128}$ G. Mitselmakher, ${ }^{128}$ L. Muniz, ${ }^{128}$ M. Park, ${ }^{128}$ R. Remington, ${ }^{128}$ A. Rinkevicius, ${ }^{128}$ M. Schmitt, ${ }^{128}$ B. Scurlock, ${ }^{128}$ P. Sellers, ${ }^{128}$ N. Skhirtladze, ${ }^{128}$ M. Snowball, ${ }^{128}$ D. Wang, ${ }^{128}$ J. Yelton, ${ }^{128}$ M. Zakaria, ${ }^{128}$ V. Gaultney, ${ }^{129}$ L. M. Lebolo, ${ }^{129}$ S. Linn, ${ }^{129}$ P. Markowitz, ${ }^{129}$ G. Martinez, ${ }^{129}$ J. L. Rodriguez, ${ }^{129}$ T. Adams, ${ }^{130}$ A. Askew ${ }^{130}$ J. Bochenek, ${ }^{130}$ J. Chen, ${ }^{130}$ B. Diamond, ${ }^{130}$ S. V. Gleyzer, ${ }^{130}$ J. Haas, ${ }^{130}$ S. Hagopian, ${ }^{130}$ V. Hagopian, ${ }^{130}$ M. Jenkins, ${ }^{130}$ K. F. Johnson, ${ }^{130}$ H. Prosper, ${ }^{130}$ S. Sekmen,,${ }^{130}$ V. Veeraraghavan, ${ }^{130}$ M. M. Baarmand, ${ }^{131}$ B. Dorney ${ }^{131}$ M. Hohlmann, ${ }^{131}$ H. Kalakhety,${ }^{131}$ I. Vodopiyanov, ${ }^{131}$ M. R. Adams, ${ }^{132}$ I. M. Anghel, ${ }^{132}$ L. Apanasevich, ${ }^{132}$ Y. Bai, ${ }^{132}$ V. E. Bazterra, ${ }^{132}$ R. R. Betts,${ }^{132}$ J. Callner, ${ }^{132}$ R. Cavanaugh, ${ }^{132}$ C. Dragoiu, ${ }^{132}$ L. Gauthier, ${ }^{132}$ C. E. Gerber, ${ }^{132}$ D. J. Hofman, ${ }^{132}$ S. Khalatyan, ${ }^{132}$ G. J. Kunde, ${ }^{132, x x}$ F. Lacroix, ${ }^{132}$ M. Malek, ${ }^{132}$ C. O'Brien, ${ }^{132}$ C. Silkworth, ${ }^{132}$ C. Silvestre, ${ }^{132}$ D. Strom, ${ }^{132}$ N. Varelas, ${ }^{132}$ U. Akgun, ${ }^{133}$ E. A. Albayrak, ${ }^{133}$ B. Bilki, ${ }^{133}$ W. Clarida, ${ }^{133}$ F. Duru, ${ }^{133}$ S. Griffiths, ${ }^{133}$ C. K. Lae, ${ }^{133}$ E. McCliment, ${ }^{133}$ J.-P. Merlo, ${ }^{133}$ H. Mermerkaya, ${ }^{133, y y}$ A. Mestvirishvili, ${ }^{133}$ A. Moeller, ${ }^{133}$ J. Nachtman, ${ }^{133}$ C. R. Newsom, ${ }^{133}$ E. Norbeck, ${ }^{133}$ J. Olson, ${ }^{133}$ Y. Onel,,${ }^{133}$ F. Ozok, ${ }^{133}$ S. Sen, ${ }^{133}$ E. Tiras, ${ }^{133}$ J. Wetzel, ${ }^{133}$ T. Yetkin, ${ }^{133}$ K. Yi, ${ }^{133}$ B. A. Barnett, ${ }^{134}$ B. Blumenfeld, ${ }^{134}$ S. Bolognesi, ${ }^{134}$ A. Bonato, ${ }^{134}$ C. Eskew, ${ }^{134}$ D. Fehling, ${ }^{134}$ G. Giurgiu, ${ }^{134}$ A. V. Gritsan, ${ }^{134}$ Z. J. Guo, ${ }^{134}$ G. Hu, ${ }^{134}$ P. Maksimovic, ${ }^{134}$ S. Rappoccio, ${ }^{134}$ M. Swartz, ${ }^{134}$ N. V. Tran, ${ }^{134}$ A. Whitbeck, ${ }^{134}$ P. Baringer, ${ }^{135}$ A. Bean, ${ }^{135}$ G. Benelli, ${ }^{135}$ O. Grachov, ${ }^{135}$ R. P. Kenny Iii, ${ }^{135}$ M. Murray, ${ }^{135}$ D. Noonan, ${ }^{135}$ S. Sanders, ${ }^{135}$ R. Stringer,${ }^{135}$ G. Tinti, ${ }^{135}$ J. S. Wood,,${ }^{135}$ V. Zhukova, ${ }^{135}$ A. F. Barfuss, ${ }^{136}$ T. Bolton, ${ }^{136}$ I. Chakaberia, ${ }^{136}$ A. Ivanov, ${ }^{136}$ S. Khalil, ${ }^{136}$ M. Makouski, ${ }^{136}$ Y. Maravin, ${ }^{136}$ S. Shrestha, ${ }^{136}$ I. Svintradze,${ }^{136}$ J. Gronberg, ${ }^{137}$ D. Lange, ${ }^{137}$ D. Wright, ${ }^{137}$ A. Baden, ${ }^{138}$ M. Boutemeur, ${ }^{138}$ B. Calvert, ${ }^{138}$ S. C. Eno, ${ }^{138}$ J. A. Gomez, ${ }^{138}$ N. J. Hadley, ${ }^{138}$ R. G. Kellogg, ${ }^{138}$ M. Kirn, ${ }^{138}$ Y. Lu, ${ }^{138}$ A. C. Mignerey, ${ }^{138}$ A. Peterman, ${ }^{138}$ K. Rossato, ${ }^{138}$ P. Rumerio, ${ }^{138}$ A. Skuja, ${ }^{138}$ J. Temple, ${ }^{138}$ M. B. Tonjes, ${ }^{138}$ S. C. Tonwar, ${ }^{138}$ E. Twedt, ${ }^{138}$ B. Alver,${ }^{139}$ G. Bauer, ${ }^{139}$ J. Bendavid, ${ }^{139}$ W. Busza, ${ }^{139}$ E. Butz, ${ }^{139}$ I. A. Cali, ${ }^{139}$ M. Chan, ${ }^{139}$ V. Dutta, ${ }^{139}$ G. Gomez Ceballos, ${ }^{139}$ M. Goncharov, ${ }^{139}$ K. A. Hahn, ${ }^{139}$ P. Harris, ${ }^{139}$ Y. Kim,,${ }^{139}$ M. Klute, ${ }^{139}$ Y.-J. Lee, ${ }^{139}$ W. Li, ${ }^{139}$ P. D. Luckey, ${ }^{139}$ T. Ma, ${ }^{139}$ S. Nahn, ${ }^{139}$ C. Paus, ${ }^{139}$ D. Ralph,${ }^{139}$ C. Roland, ${ }^{139}$ G. Roland,${ }^{139}$ M. Rudolph, ${ }^{139}$ G. S. F. Stephans,${ }^{139}$ F. Stöckli, ${ }^{139}$ K. Sumorok, ${ }^{139}$ K. Sung, ${ }^{139}$ D. Velicanu, ${ }^{139}$ E. A. Wenger, ${ }^{139}$ R. Wolf, ${ }^{139}$ B. Wyslouch, ${ }^{139}$ S. Xie, ${ }^{139}$ M. Yang, ${ }^{139}$ Y. Yilmaz, ${ }^{139}$ A. S. Yoon, ${ }^{139}$ M. Zanetti, ${ }^{139}$ S. I. Cooper, ${ }^{140}$ P. Cushman, ${ }^{140}$ B. Dahmes,,${ }^{140}$

A. De Benedetti, ${ }^{140}$ G. Franzoni, ${ }^{140}$ A. Gude, ${ }^{140}$ J. Haupt,${ }^{140}$ K. Klapoetke, ${ }^{140}$ Y. Kubota, ${ }^{140}$ J. Mans,${ }^{140}$ N. Pastika, ${ }^{140}$ V. Rekovic, ${ }^{140}$ R. Rusack, ${ }^{140}$ M. Sasseville, ${ }^{140}$ A. Singovsky, ${ }^{140}$ N. Tambe, ${ }^{140}$ J. Turkewitz, ${ }^{140}$ L. M. Cremaldi, ${ }^{141}$ R. Godang, ${ }^{141}$ R. Kroeger, ${ }^{141}$ L. Perera, ${ }^{141}$ R. Rahmat, ${ }^{141}$ D. A. Sanders, ${ }^{141}$ D. Summers, ${ }^{141}$ E. Avdeeva, ${ }^{142}$ K. Bloom, ${ }^{142}$ S. Bose,${ }^{142}$ J. Butt ${ }^{142}$ D. R. Claes, ${ }^{142}$ A. Dominguez,${ }^{142}$ M. Eads, ${ }^{142}$ P. Jindal, ${ }^{142}$ J. Keller, ${ }^{142}$ I. Kravchenko, ${ }^{142}$ J. Lazo-Flores, ${ }^{142}$ H. Malbouisson, ${ }^{142}$ S. Malik, ${ }^{142}$ G. R. Snow, ${ }^{142}$ U. Baur, ${ }^{143}$ A. Godshalk, ${ }^{143}$ I. Iashvili, ${ }^{143}$ S. Jain, ${ }^{143}$ A. Kharchilava, ${ }^{143}$ A. Kumar, ${ }^{143}$ K. Smith, ${ }^{143}$ Z. Wan, ${ }^{143}$ G. Alverson, ${ }^{144}$ E. Barberis ${ }^{144}$ D. Baumgartel, ${ }^{144}$ M. Chasco,${ }^{144}$ D. Trocino, ${ }^{144}$ D. Wood,${ }^{144}$ J. Zhang, ${ }^{144}$ A. Anastassov, ${ }^{145}$ A. Kubik, ${ }^{145}$ N. Mucia, ${ }^{145}$ N. Odell, ${ }^{145}$ R. A. Ofierzynski, ${ }^{145}$ B. Pollack, ${ }^{145}$ A. Pozdnyakov,${ }^{145}$ M. Schmitt, ${ }^{145}$ S. Stoynev, ${ }^{145}$ M. Velasco, ${ }^{145}$ S. Won, ${ }^{145}$ L. Antonelli, ${ }^{146}$ D. Berry, ${ }^{146}$ A. Brinkerhoff, ${ }^{146}$ M. Hildreth, ${ }^{146}$ C. Jessop, ${ }^{146}$ D. J. Karmgard, ${ }^{146}$ J. Kolb, ${ }^{146}$ T. Kolberg, ${ }^{146}$ K. Lannon, ${ }^{146}$ W. Luo, ${ }^{146}$ S. Lynch, ${ }^{146}$ N. Marinelli, ${ }^{146}$ 
D. M. Morse, ${ }^{146}$ T. Pearson, ${ }^{146}$ R. Ruchti, ${ }^{146}$ J. Slaunwhite, ${ }^{146}$ N. Valls, ${ }^{146}$ M. Wayne, ${ }^{146}$ J. Ziegler, ${ }^{146}$ B. Bylsma, ${ }^{147}$ L. S. Durkin, ${ }^{147}$ C. Hill, ${ }^{147}$ P. Killewald,${ }^{147}$ K. Kotov, ${ }^{147}$ T. Y. Ling, ${ }^{147}$ M. Rodenburg, ${ }^{147}$ C. Vuosalo, ${ }^{147}$ G. Williams, ${ }^{147}$ N. Adam, ${ }^{148}$ E. Berry, ${ }^{148}$ P. Elmer, ${ }^{148}$ D. Gerbaudo, ${ }^{148}$ V. Halyo, ${ }^{148}$ P. Hebda, ${ }^{148}$ A. Hunt,${ }^{148}$ E. Laird, ${ }^{148}$ D. Lopes Pegna, ${ }^{148}$ P. Lujan, ${ }^{148}$ D. Marlow, ${ }^{148}$ T. Medvedeva, ${ }^{148}$ M. Mooney, ${ }^{148}$ J. Olsen, ${ }^{148}$ P. Piroué, ${ }^{148}$ X. Quan, ${ }^{148}$ A. Raval, ${ }^{148}$ H. Saka, ${ }^{148}$ D. Stickland, ${ }^{148}$ C. Tully, ${ }^{148}$ J. S. Werner, ${ }^{148}$ A. Zuranski, ${ }^{148}$ J. G. Acosta, ${ }^{149}$ X. T. Huang, ${ }^{149}$ A. Lopez ${ }^{149}$ H. Mendez ${ }^{149}$ S. Oliveros ${ }^{149}$ J. E. Ramirez Vargas, ${ }^{149}$ A. Zatserklyaniy, ${ }^{149}$ E. Alagoz, ${ }^{150}$ V.E. Barnes,${ }^{150}$ D. Benedetti, ${ }^{150}$ G. Bolla, ${ }^{150}$ L. Borrello, ${ }^{150}$ D. Bortoletto, ${ }^{150}$ M. De Mattia, ${ }^{150}$ A. Everett, ${ }^{150}$ L. Gutay, ${ }^{150}$ Z. Hu, ${ }^{150}$ M. Jones, ${ }^{150}$ O. Koybasi, ${ }^{150}$ M. Kress, ${ }^{150}$ A. T. Laasanen, ${ }^{150}$ N. Leonardo, ${ }^{150}$ V. Maroussov, ${ }^{150}$ P. Merkel,,${ }^{150}$ D. H. Miller,${ }^{150}$ N. Neumeister,${ }^{150}$ I. Shipsey, ${ }^{150}$ D. Silvers, ${ }^{150}$ A. Svyatkovskiy, ${ }^{150}$ M. Vidal Marono, ${ }^{150}$ H. D. Yoo, ${ }^{150}$ J. Zablocki,${ }^{150}$ Y. Zheng, ${ }^{150}$ S. Guragain, ${ }^{151}$ N. Parashar, ${ }^{151}$ A. Adair, ${ }^{152}$ C. Boulahouache, ${ }^{152}$ V. Cuplov, ${ }^{152}$ K. M. Ecklund, ${ }^{152}$ F. J. M. Geurts, ${ }^{152}$ B. P. Padley, ${ }^{152}$ R. Redjimi, ${ }^{152}$ J. Roberts, ${ }^{152}$ J. Zabel, ${ }^{152}$ B. Betchart, ${ }^{153}$ A. Bodek, ${ }^{153}$ Y. S. Chung, ${ }^{153}$ R. Covarelli, ${ }^{153}$ P. de Barbaro, ${ }^{153}$ R. Demina, ${ }^{153}$ Y. Eshaq,${ }^{153}$ H. Flacher, ${ }^{153}$ A. Garcia-Bellido, ${ }^{153}$ P. Goldenzweig, ${ }^{153}$ Y. Gotra, ${ }^{153}$ J. Han,${ }^{153}$ A. Harel, ${ }^{153}$ D. C. Miner, ${ }^{153}$ G. Petrillo, ${ }^{153}$ W. Sakumoto, ${ }^{153}$ D. Vishnevskiy, ${ }^{153}$ M. Zielinski, ${ }^{153}$ A. Bhatti, ${ }^{154}$ R. Ciesielski, ${ }^{154}$ L. Demortier, ${ }^{154}$ K. Goulianos, ${ }^{154}$ G. Lungu, ${ }^{154}$ S. Malik, ${ }^{154}$ C. Mesropian, ${ }^{154}$ S. Arora,${ }^{155}$ O. Atramentov, ${ }^{155}$ A. Barker ${ }^{155}$ J. P. Chou, ${ }^{155}$ C. Contreras-Campana, ${ }^{155}$ E. Contreras-Campana, ${ }^{155}$ D. Duggan, ${ }^{155}$ D. Ferencek, ${ }^{155}$ Y. Gershtein, ${ }^{155}$ R. Gray, ${ }^{155}$ E. Halkiadakis, ${ }^{155}$ D. Hidas, ${ }^{155}$ D. Hits, ${ }^{155}$ A. Lath, ${ }^{155}$ S. Panwalkar,${ }^{155}$ M. Park,${ }^{155}$ R. Patel,${ }^{155}$ A. Richards, ${ }^{155}$ K. Rose,${ }^{155}$ S. Salur,${ }^{155}$ S. Schnetzer ${ }^{155}$ S. Somalwar, ${ }^{155}$ R. Stone,${ }^{155}$ S. Thomas, ${ }^{155}$ G. Cerizza, ${ }^{156}$ M. Hollingsworth, ${ }^{156}$ S. Spanier ${ }^{156}$ Z. C. Yang,${ }^{156}$ A. York, ${ }^{156}$ R. Eusebi,${ }^{157}$ W. Flanagan,${ }^{157}$ J. Gilmore,${ }^{157}$ T. Kamon, ${ }^{157, z z}$ V. Khotilovich, ${ }^{157}$ R. Montalvo, ${ }^{157}$ I. Osipenkov, ${ }^{157}$ Y. Pakhotin, ${ }^{157}$ A. Perloff, ${ }^{157}$ J. Roe, ${ }^{157}$ A. Safonov, ${ }^{157}$ S. Sengupta, ${ }^{157}$ I. Suarez,${ }^{157}$ A. Tatarinov, ${ }^{157}$ D. Toback, ${ }^{157}$ N. Akchurin, ${ }^{158}$ C. Bardak,${ }^{158}$ J. Damgov, ${ }^{158}$ P. R. Dudero, ${ }^{158}$ C. Jeong, ${ }^{158}$ K. Kovitanggoon, ${ }^{158}$ S. W. Lee, ${ }^{158}$ T. Libeiro, ${ }^{158}$ P. Mane,${ }^{158}$ Y. Roh,${ }^{158}$ A. Sill, ${ }^{158}$ I. Volobouev, ${ }^{158}$ R. Wigmans, ${ }^{158}$ E. Yazgan, ${ }^{158}$ E. Appelt, ${ }^{159}$ E. Brownson, ${ }^{159}$ D. Engh, ${ }^{159}$ C. Florez, ${ }^{159}$ W. Gabella, ${ }^{159}$ A. Gurrola, ${ }^{159}$ M. Issah, ${ }^{159}$ W. Johns, ${ }^{159}$ C. Johnston, ${ }^{159}$ P. Kurt, ${ }^{159}$ C. Maguire, ${ }^{159}$ A. Melo,,${ }^{159}$ P. Sheldon, ${ }^{159}$ B. Snook, ${ }^{159}$ S. Tuo, ${ }^{159}$ J. Velkovska, ${ }^{159}$ M. W. Arenton, ${ }^{160}$ M. Balazs, ${ }^{160}$ S. Boutle, ${ }^{160}$ S. Conetti, ${ }^{160}$ B. Cox ${ }^{160}$ B. Francis ${ }^{160}$ S. Goadhouse, ${ }^{160}$ J. Goodell, ${ }^{160}$ R. Hirosky, ${ }^{160}$ A. Ledovskoy, ${ }^{160}$ C. Lin, ${ }^{160}$ C. Neu, ${ }^{160}$ J. Wood, ${ }^{160}$ R. Yohay, ${ }^{160}$ S. Gollapinni, ${ }^{161}$ R. Harr, ${ }^{161}$ P. E. Karchin, ${ }^{161}$ C. Kottachchi Kankanamge Don, ${ }^{161}$ P. Lamichhane, ${ }^{161}$ M. Mattson, ${ }^{161}$ C. Milstène, ${ }^{161}$ A. Sakharov, ${ }^{161}$ M. Anderson, ${ }^{162}$ M. Bachtis, ${ }^{162}$ D. Belknap ${ }^{162}$ J. N. Bellinger, ${ }^{162}$ J. Bernardini, ${ }^{162}$ D. Carlsmith, ${ }^{162}$ M. Cepeda, ${ }^{162}$ S. Dasu, ${ }^{162}$ J. Efron, ${ }^{162}$ E. Friis, ${ }^{162}$ L. Gray, ${ }^{162}$ K. S. Grogg, ${ }^{162}$ M. Grothe, ${ }^{162}$ R. Hall-Wilton, ${ }^{162}$ M. Herndon, ${ }^{162}$ A. Hervé, ${ }^{162}$ P. Klabbers, ${ }^{162}$ J. Klukas, ${ }^{162}$ A. Lanaro, ${ }^{162}$ C. Lazaridis, ${ }^{162}$ J. Leonard, ${ }^{162}$ R. Loveless, ${ }^{162}$ A. Mohapatra, ${ }^{162}$ I. Ojalvo, ${ }^{162}$ G. A. Pierro, ${ }^{162}$ I. Ross, ${ }^{162}$ A. Savin, ${ }^{162}$ W. H. Smith, ${ }^{162}$ J. Swanson, ${ }^{162}$ and M. Weinberg ${ }^{162}$

\title{
(CMS Collaboration)
}

\author{
${ }^{1}$ Yerevan Physics Institute, Yerevan, Armenia \\ ${ }^{2}$ Institut für Hochenergiephysik der OeAW, Wien, Austria \\ ${ }^{3}$ National Centre for Particle and High Energy Physics, Minsk, Belarus \\ ${ }^{4}$ Universiteit Antwerpen, Antwerpen, Belgium \\ ${ }^{5}$ Vrije Universiteit Brussel, Brussel, Belgium \\ ${ }^{6}$ Université Libre de Bruxelles, Bruxelles, Belgium \\ ${ }^{7}$ Ghent University, Ghent, Belgium \\ ${ }^{8}$ Université Catholique de Louvain, Louvain-la-Neuve, Belgium \\ ${ }^{9}$ Université de Mons, Mons, Belgium \\ ${ }^{10}$ Centro Brasileiro de Pesquisas Fisicas, Rio de Janeiro, Brazil \\ ${ }^{11}$ Universidade do Estado do Rio de Janeiro, Rio de Janeiro, Brazil \\ ${ }^{12}$ Instituto de Fisica Teorica, Universidade Estadual Paulista, Sao Paulo, Brazil \\ ${ }^{13}$ Institute for Nuclear Research and Nuclear Energy, Sofia, Bulgaria \\ ${ }^{14}$ University of Sofia, Sofia, Bulgaria \\ ${ }^{15}$ Institute of High Energy Physics, Beijing, China \\ ${ }^{16}$ State Key Laboratory of Nuclear Physics and Technology, Peking University, Beijing, China \\ ${ }^{17}$ Universidad de Los Andes, Bogota, Colombia \\ ${ }^{18}$ Technical University of Split, Split, Croatia \\ ${ }^{19}$ University of Split, Split, Croatia
}




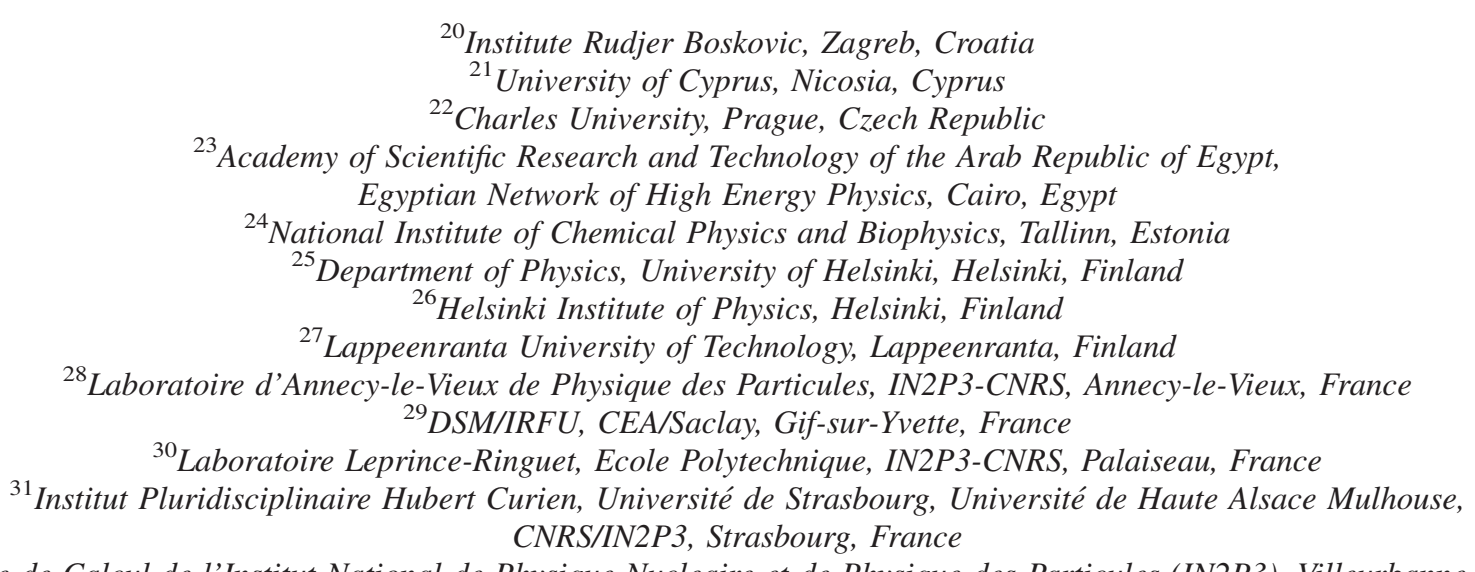

${ }^{32}$ Centre de Calcul de l'Institut National de Physique Nucleaire et de Physique des Particules (IN2P3), Villeurbanne, France

${ }^{33}$ Université de Lyon, Université Claude Bernard Lyon 1, CNRS-IN2P3, Institut de Physique Nucléaire de Lyon, Villeurbanne, France

${ }^{34}$ Institute of High Energy Physics and Informatization, Tbilisi State University, Tbilisi, Georgia

${ }^{35}$ RWTH Aachen University, I. Physikalisches Institut, Aachen, Germany

${ }^{36}$ RWTH Aachen University, III. Physikalisches Institut A, Aachen, Germany

${ }^{37}$ RWTH Aachen University, III. Physikalisches Institut B, Aachen, Germany

${ }^{38}$ Deutsches Elektronen-Synchrotron, Hamburg, Germany

${ }^{39}$ University of Hamburg, Hamburg, Germany

${ }^{40}$ Institut für Experimentelle Kernphysik, Karlsruhe, Germany

${ }^{41}$ Institute of Nuclear Physics “Demokritos,” Aghia Paraskevi, Greece

${ }^{42}$ University of Athens, Athens, Greece

${ }^{43}$ University of Ioánnina, Ioánnina, Greece

${ }^{44}$ KFKI Research Institute for Particle and Nuclear Physics, Budapest, Hungary

${ }^{45}$ Institute of Nuclear Research ATOMKI, Debrecen, Hungary

${ }^{46}$ University of Debrecen, Debrecen, Hungary

${ }^{47}$ Panjab University, Chandigarh, India

${ }^{48}$ University of Delhi, Delhi, India

${ }^{49}$ Saha Institute of Nuclear Physics, Kolkata, India

${ }^{50}$ Bhabha Atomic Research Centre, Mumbai, India

${ }^{51}$ Tata Institute of Fundamental Research-EHEP, Mumbai, India

${ }^{52}$ Tata Institute of Fundamental Research-HECR, Mumbai, India

${ }^{53}$ Institute for Research and Fundamental Sciences (IPM), Tehran, Iran

${ }^{54 a}$ INFN Sezione di Bari, Bari, Italy

${ }^{54 \mathrm{~b}}$ Università di Bari, Bari, Italy

${ }^{54 \mathrm{c}}$ Politecnico di Bari, Bari, Italy

${ }^{55}$ INFN Sezione di Bologna, Bologna, Italy

${ }^{55 \mathrm{~b}}$ Università di Bologna, Bologna, Italy

${ }^{56 a}$ INFN Sezione di Catania, Catania, Italy

${ }^{56 \mathrm{~b}}$ Università di Catania, Catania, Italy

${ }^{57 a}$ INFN Sezione di Firenze, Firenze, Italy

${ }^{57 \mathrm{~b}}$ Università di Firenze, Firenze, Italy

${ }^{58}$ INFN Laboratori Nazionali di Frascati, Frascati, Italy

${ }^{59}$ INFN Sezione di Genova, Genova, Italy

${ }^{60 \mathrm{a}}$ INFN Sezione di Milano-Bicocca, Milano, Italy

${ }^{60 \mathrm{~b}}$ Università di Milano-Bicocca, Milano, Italy

${ }^{61 \mathrm{a}}$ INFN Sezione di Napoli, Napoli, Italy

${ }^{61 \mathrm{~b}}$ Università di Napoli "Federico II," Napoli, Italy

${ }^{62 a}$ INFN Sezione di Padova, Padova, Italy

${ }^{62 \mathrm{~b}}$ Università di Padova, Padova, Italy

${ }^{62 \mathrm{c}}$ Università di Trento (Trento), Padova, Italy

${ }^{63}$ INFN Sezione di Pavia, Pavia, Italy

${ }^{63 \mathrm{~b}}$ Università di Pavia, Pavia, Italy

${ }^{64 a}$ INFN Sezione di Perugia, Italy

${ }^{64 \mathrm{~b}}$ Università di Perugia, Italy

${ }^{65 a}$ INFN Sezione di Pisa, Pisa, Italy

${ }^{65 \mathrm{~b}}$ Università di Pisa, Pisa, Italy

${ }^{65 \mathrm{c} S c u o l a}$ Normale Superiore di Pisa, Pisa, Italy 
${ }^{66 a}$ INFN Sezione di Roma, Roma, Italy

${ }^{66 \mathrm{~b}}$ Università di Roma “La Sapienza," Roma, Italy

${ }^{67 a}$ INFN Sezione di Torino, Torino, Italy

${ }^{67 \mathrm{~b}}$ Università di Torino, Torino, Italy

${ }^{67 \mathrm{c}}$ Università del Piemonte Orientale (Novara), Torino, Italy

${ }^{68 \mathrm{a}}$ INFN Sezione di Trieste, Trieste, Italy

${ }^{68 \mathrm{~b}}$ Università di Trieste, Trieste, Italy

${ }^{69}$ Kangwon National University, Chunchon, Korea

${ }^{70}$ Kyungpook National University, Daegu, Korea

${ }^{71}$ Chonnam National University, Institute for Universe and Elementary Particles, Kwangju, Korea

${ }^{72}$ Konkuk University, Seoul, Korea

${ }^{73}$ Korea University, Seoul, Korea

${ }^{74}$ University of Seoul, Seoul, Korea

${ }^{75}$ Sungkyunkwan University, Suwon, Korea

${ }^{76}$ Vilnius University, Vilnius, Lithuania

${ }^{77}$ Centro de Investigacion y de Estudios Avanzados del IPN, Mexico City, Mexico

${ }^{78}$ Universidad Iberoamericana, Mexico City, Mexico

${ }^{79}$ Benemerita Universidad Autonoma de Puebla, Puebla, Mexico

${ }^{80}$ Universidad Autónoma de San Luis Potosí, San Luis Potosí, Mexico

${ }^{81}$ University of Auckland, Auckland, New Zealand

${ }^{82}$ University of Canterbury, Christchurch, New Zealand

${ }^{83}$ National Centre for Physics, Quaid-I-Azam University, Islamabad, Pakistan

${ }^{84}$ Institute of Experimental Physics, Faculty of Physics, University of Warsaw, Warsaw, Poland

${ }^{85}$ Soltan Institute for Nuclear Studies, Warsaw, Poland

${ }^{86}$ Laboratório de Instrumentação e Física Experimental de Partículas, Lisboa, Portugal

${ }^{87}$ Joint Institute for Nuclear Research, Dubna, Russia

${ }^{88}$ Petersburg Nuclear Physics Institute, Gatchina (St Petersburg), Russia

${ }^{89}$ Institute for Nuclear Research, Moscow, Russia

${ }^{90}$ Institute for Theoretical and Experimental Physics, Moscow, Russia

${ }^{91}$ Moscow State University, Moscow, Russia

${ }^{92}$ P. N. Lebedev Physical Institute, Moscow, Russia

${ }^{93}$ State Research Center of Russian Federation, Institute for High Energy Physics, Protvino, Russia

${ }^{94}$ University of Belgrade, Faculty of Physics and Vinca Institute of Nuclear Sciences, Belgrade, Serbia

${ }^{95}$ Centro de Investigaciones Energéticas Medioambientales y Tecnológicas (CIEMAT), Madrid, Spain

${ }^{96}$ Universidad Autónoma de Madrid, Madrid, Spain

${ }^{97}$ Universidad de Oviedo, Oviedo, Spain

${ }^{98}$ Instituto de Física de Cantabria (IFCA), CSIC-Universidad de Cantabria, Santander, Spain

${ }^{99}$ CERN, European Organization for Nuclear Research, Geneva, Switzerland

${ }^{100}$ Paul Scherrer Institut, Villigen, Switzerland

${ }^{101}$ Institute for Particle Physics, ETH Zurich, Zurich, Switzerland

${ }^{102}$ Universität Zürich, Zurich, Switzerland

${ }^{103}$ National Central University, Chung-Li, Taiwan

${ }^{104}$ National Taiwan University (NTU), Taipei, Taiwan

${ }^{105}$ Cukurova University, Adana, Turkey

${ }^{106}$ Middle East Technical University, Physics Department, Ankara, Turkey

${ }^{107}$ Bogazici University, Istanbul, Turkey

${ }^{108}$ National Scientific Center, Kharkov Institute of Physics and Technology, Kharkov, Ukraine

${ }^{109}$ University of Bristol, Bristol, United Kingdom

${ }^{110}$ Rutherford Appleton Laboratory, Didcot, United Kingdom

${ }^{111}$ Imperial College, London, United Kingdom

${ }^{112}$ Brunel University, Uxbridge, United Kingdom

${ }^{113}$ Baylor University, Waco, Texas, USA

${ }^{114}$ The University of Alabama, Tuscaloosa, Alabama, USA

${ }^{115}$ Boston University, Boston, Massachusetts, USA

${ }^{116}$ Brown University, Providence, Rhode Island, USA

${ }^{117}$ University of California, Davis, Davis, California, USA

${ }^{118}$ University of California, Los Angeles, Los Angeles, California, USA

${ }^{119}$ University of California, Riverside, Riverside, California, USA

${ }^{120}$ University of California, San Diego, La Jolla, California, USA

${ }^{121}$ University of California, Santa Barbara, Santa Barbara, California, USA

${ }^{122}$ California Institute of Technology, Pasadena, California, USA 
${ }^{123}$ Carnegie Mellon University, Pittsburgh, Pennsylvania, USA

${ }^{124}$ University of Colorado at Boulder, Boulder, Colorado, USA

${ }^{125}$ Cornell University, Ithaca, New York, USA

${ }^{126}$ Fairfield University, Fairfield, Connecticut, USA

${ }^{127}$ Fermi National Accelerator Laboratory, Batavia, Illinois, USA

${ }^{128}$ University of Florida, Gainesville, Florida, USA

${ }^{129}$ Florida International University, Miami, Florida, USA

${ }^{130}$ Florida State University, Tallahassee, Florida, USA

${ }^{131}$ Florida Institute of Technology, Melbourne, Florida, USA

${ }^{132}$ University of Illinois at Chicago (UIC), Chicago, Illinois, USA

${ }^{133}$ The University of Iowa, Iowa City, Iowa, USA

${ }^{134}$ Johns Hopkins University, Baltimore, Maryland, USA

${ }^{135}$ The University of Kansas, Lawrence, Kansas, USA

${ }^{136}$ Kansas State University, Manhattan, Kansas, USA

${ }^{137}$ Lawrence Livermore National Laboratory, Livermore, California, USA

${ }^{138}$ University of Maryland, College Park, Maryland, USA

${ }^{139}$ Massachusetts Institute of Technology, Cambridge, Massachusetts, USA

${ }^{140}$ University of Minnesota, Minneapolis, Minnesota, USA

${ }^{141}$ University of Mississippi, University, Mississippi, USA

${ }^{142}$ University of Nebraska-Lincoln, Lincoln, Nebraska, USA

${ }^{143}$ State University of New York at Buffalo, Buffalo, New York, USA

${ }^{144}$ Northeastern University, Boston, Massachusetts, USA

${ }^{145}$ Northwestern University, Evanston, Illinois, USA

${ }^{146}$ University of Notre Dame, Notre Dame, Indiana, USA

${ }^{147}$ The Ohio State University, Columbus, Ohio, USA

${ }^{148}$ Princeton University, Princeton, New Jersey, USA

${ }^{149}$ University of Puerto Rico, Mayaguez, Puerto Rico

${ }^{150}$ Purdue University, West Lafayette, Indiana, USA

${ }^{151}$ Purdue University Calumet, Hammond, Indiana, USA

${ }^{152}$ Rice University, Houston, Texas, USA

${ }^{153}$ University of Rochester, Rochester, New York, USA

${ }^{154}$ The Rockefeller University, New York, New York, USA

${ }^{155}$ Rutgers, the State University of New Jersey, Piscataway, New Jersey, USA

${ }^{156}$ University of Tennessee, Knoxville, Tennessee, USA

${ }^{157}$ Texas A\&M University, College Station, Texas, USA

${ }^{158}$ Texas Tech University, Lubbock, Texas, USA

${ }^{159}$ Vanderbilt University, Nashville, Tennessee, USA

${ }^{160}$ University of Virginia, Charlottesville, Virginia, USA

${ }^{161}$ Wayne State University, Detroit, Michigan, USA

${ }^{162}$ University of Wisconsin, Madison, Wisconsin, USA

${ }^{\mathrm{a} D e c e a s e d .}$

${ }^{\mathrm{b}}$ Also at CERN, European Organization for Nuclear Research, Geneva, Switzerland.

${ }^{c}$ Also at National Institute of Chemical Physics and Biophysics, Tallinn, Estonia.

${ }^{\mathrm{d}}$ Also at Universidade Federal do ABC, Santo Andre, Brazil.

${ }^{\mathrm{e}}$ Also at California Institute of Technology, Pasadena, California, USA.

${ }_{\mathrm{f}}^{\mathrm{f}}$ Also at Laboratoire Leprince-Ringuet, Ecole Polytechnique, IN2P3-CNRS, Palaiseau, France.

${ }^{\mathrm{g}}$ Also at Suez Canal University, Suez, Egypt.

${ }^{\mathrm{h}}$ Also at Cairo University, Cairo, Egypt.

${ }^{\mathrm{i}}$ Also at British University, Cairo, Egypt.

${ }^{\mathrm{j}}$ Also at Fayoum University, El-Fayoum, Egypt.

${ }^{\mathrm{k}}$ Also at Ain Shams University, Cairo, Egypt.

${ }^{1}$ Also at Soltan Institute for Nuclear Studies, Warsaw, Poland.

${ }^{\mathrm{m}}$ Also at Université de Haute-Alsace, Mulhouse, France.

${ }^{\mathrm{n}}$ Also at Moscow State University, Moscow, Russia.

${ }^{\circ}$ Also at Brandenburg University of Technology, Cottbus, Germany.

${ }^{\mathrm{p}}$ Also at Institute of Nuclear Research ATOMKI, Debrecen, Hungary.

${ }^{\mathrm{q}}$ Also at Eötvös Loránd University, Budapest, Hungary.

${ }^{\mathrm{r}}$ Also at Tata Institute of Fundamental Research-HECR, Mumbai, India. 
${ }^{\mathrm{s}}$ Also at University of Visva-Bharati, Santiniketan, India.

${ }^{t}$ Also at Sharif University of Technology, Tehran, Iran.

${ }^{\mathrm{u}}$ Also at Isfahan University of Technology, Isfahan, Iran.

${ }^{v}$ Also at Shiraz University, Shiraz, Iran.

${ }^{w}$ Also at Plasma Physics Research Center, Science and Research Branch, Islamic Azad University, Teheran, Iran.

${ }^{x}$ Also at Facoltà Ingegneria Università di Roma, Roma, Italy.

${ }^{y}$ Also at Università della Basilicata, Potenza, Italy.

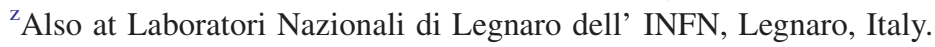

${ }^{\text {aa } A l s o ~ a t ~ U n i v e r s i t a ̀ ~ d e g l i ~ s t u d i ~ d i ~ S i e n a, ~ S i e n a, ~ I t a l y . ~}$

${ }^{\mathrm{bb}}$ Also at Faculty of Physics of University of Belgrade, Belgrade, Serbia.

${ }^{\mathrm{cc}}$ Also at University of California, Los Angeles, Los Angeles, California, USA.

${ }^{\mathrm{dd}}$ Also at University of Florida, Gainesville, Florida, USA.

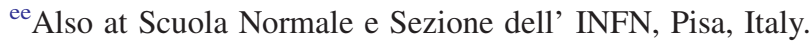

${ }^{\mathrm{ff}}$ Also at INFN Sezione di Roma, Università di Roma "La Sapienza," Roma, Italy.

${ }^{\mathrm{gg}}$ Also at University of Athens, Athens, Greece.

${ }^{\mathrm{hh}}$ Also at Rutherford Appleton Laboratory, Didcot, United Kingdom.

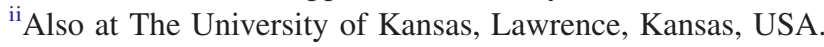

${ }^{\mathrm{jj}} \mathrm{Also}$ at University of Belgrade, Faculty of Physics and Vinca Institute of Nuclear Sciences, Belgrade, Serbia.

${ }^{\mathrm{kk}}$ Also at Paul Scherrer Institut, Villigen, Switzerland.

${ }^{11}$ Also at Institute for Theoretical and Experimental Physics, Moscow, Russia.

${ }^{\mathrm{mm}}$ Also at Gaziosmanpasa University, Tokat, Turkey.

${ }^{\mathrm{nn}}$ Also at Adiyaman University, Adiyaman, Turkey.

${ }^{\mathrm{oo}}$ Also at The University of Iowa, Iowa City, Iowa, USA.

ppAlso at Mersin University, Mersin, Turkey.

${ }^{\mathrm{qq}} \mathrm{Also}$ at Kafkas University, Kars, Turkey.

${ }^{\mathrm{rr}}$ Also at Suleyman Demirel University, Isparta, Turkey.

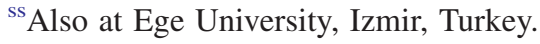

${ }^{t t}$ Also at School of Physics and Astronomy, University of Southampton, Southampton, United Kingdom.

uu Also at INFN Sezione di Perugia, Università di Perugia, Perugia, Italy.

${ }^{\mathrm{vv}}$ Also at Utah Valley University, Orem, Utah, USA.

${ }^{w w}$ Also at Institute for Nuclear Research, Moscow, Russia.

${ }^{\mathrm{xx}}$ Also at Los Alamos National Laboratory, Los Alamos, New Mexico, USA.

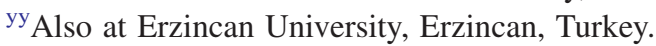

${ }^{z z}$ Also at Kyungpook National University, Daegu, Korea. 